\title{
Die Anodenvorgänge bei der Elektrolyse von in Kryolith gelöster Tonerde. I .
}

\author{
Paul Drossbach und Tomoyasu Hashino \\ Aus dem Institut für Physikalische Chemie und Elektrochemie der \\ Technischen Hochschule München
}

Received Oct. 2, 1965

\begin{abstract}
A study was made on the application of the galvanostatic method for the determination of the anodic process in electrolysis of molten $\mathrm{Li}_{3} \mathrm{AlF}_{6}-\mathrm{Na}_{3} \mathrm{AlF}_{6}-\mathrm{Al}_{2} \mathrm{O}_{3}$ systems. All possible mass transfer processes in the electrolyte and systems of heterogeneous single chemical reactions on the graphite anode were discussed theoretically and these calculated polarisation potentials were examined by the observed potential-time curves at different current densities. Anodic polarisation potential obtained for alumina dissolved in a molten cryolite mixture at $870^{\circ} \mathrm{C}$ represented (1) mass transfer process with convection of $\mathrm{O}^{2-}$-ions or radical ions, each of which contains only one oxygen atom, (2) following series of heterogeneous reactions:
\end{abstract}

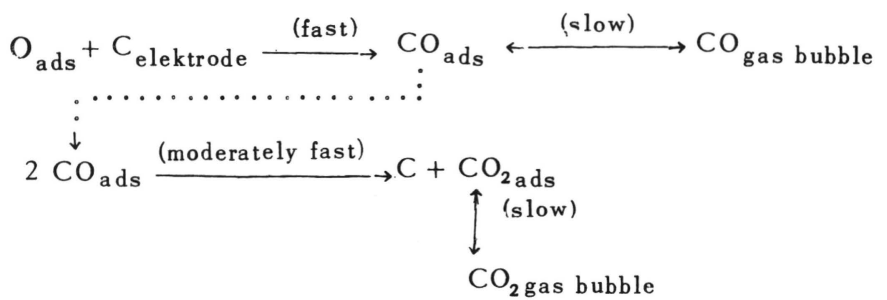

and (3) succeeding ohmic drop on the electrode caused by gas film or gas bubbles.

\section{Einleitung}

Zahllose Untersuchungen über die Elektrolyse von in Kryolith gelöster Tonerde werden selbstverständig wegen der werden. Bedeutung für die industrielle Gewinnung von Aluniniummetall seit langem durchgeführt, infolgedessen werden verschiedene Theorien über die sog. Elektrodenvorgänge berichtet, die zum großen Teil aus den sta- tionären Meßmethoden abgeleitet werden. ${ }^{1) 2)}$

Zur Messung der Potential - Zeit-Kurven bei konstanter Stromstärke diente die Schmelzflußelektrolyse von $\mathrm{Li}_{3} \mathrm{AlF}_{6}(35 \mathrm{Mo1 \%})$ $-\mathrm{Na}_{3} \mathrm{AlF}_{6}(65 \mathrm{Mo1} \%)-\mathrm{Al}_{2} \mathrm{O}_{3}(5 \mathrm{Mo} 1 \%)$ bei $870^{\circ} \mathrm{C}$, über die schon frühr berichtet wurde.3)4)

In den verschiedenen bis $\mathrm{jet} z \mathrm{t}$ gebrachten Veröffentlichungen werden für die tatsächlichen nichtstationären Elektrodenvorgänge keine zuverlässigen Ergebnisse über die Dissoziation der Tonerde gebracht, 
sondern das Dissoziationsscheme je nach der Meßmethode angesetzt. ${ }^{4) 5(6) 7)}$. Es steht aber fest, daB $\mathrm{CO}$ bzw. $\mathrm{CO}_{2}$ als Endprodukte der Anodenprozesse gebildet werden, weshalb den Modellen für unsere mathematische Behandlung nur diese Tatsache zugrunde liegt, d. h. alle theoretisch möglichen Prozesse von Tonerde zu CO bzw. $\mathrm{CO}_{2}$ sind Gegenstände dieser Untersuchung.

Zur Bestimmung der Diffusionskonstante wird gewöhnlich oft die Grenzstromdichte gemessen. Bei unserem Elektrolyt tritt jedoch die Schwierigkeit auf, daß in der Nähe des Grenzstromes der Anodeneffekt auftritt und die genaue Messung des Grenzstromes und des Grenzpotentials sehr erschwert. Da der bei konstanter Stromdichte erhaltene zeitliche Diffusionspolarisations verlauf mit Konvektion aber durch die Voraussetzung einer Dicke der Nernstschen Diffusionsschicht berechnet werden kann, ist es sicher möglich, unter der Versuchsbedingung ohne Anodeneffekt den wahrscheinlichsten Vorgang auszuwählen, aus dem ein nicht abnormaler Wert für sowohl Diffusionskonstante als auch Dicke der Stromdichte sich ergeben soll.

Die theoretischen Gleichungen für die Reaktionspolarisation wurde unter der Annahme verschiedener Möglichkeit der Elektrodenreaktionsfolgen erhalten. Bei ihrer praktischen Anwendung war jedoch sehr hinderlich, daß die Werte der darin benutzten Geschwindigkeitskonstanten zum großen Teil noch nicht bekannt sind. Durch die Diskussion der Funktion aber wurde die Auswahl des wahrscheinlichsten Prozesses schließlich ermöglicht.

\section{Theoretische Betrachtung}

Um die Anodenprozesse bei der Elektrolyse von in Kryolith gelöster Tonerde verständlich zu machen, kann man wie bei der
Wasserstoffentwicklung in wäßriger Lösung ${ }^{8)}$ die in Abb. 1 dargestellten nacheinander verlaufenden Vorgänge unterscheicen, nämilich.

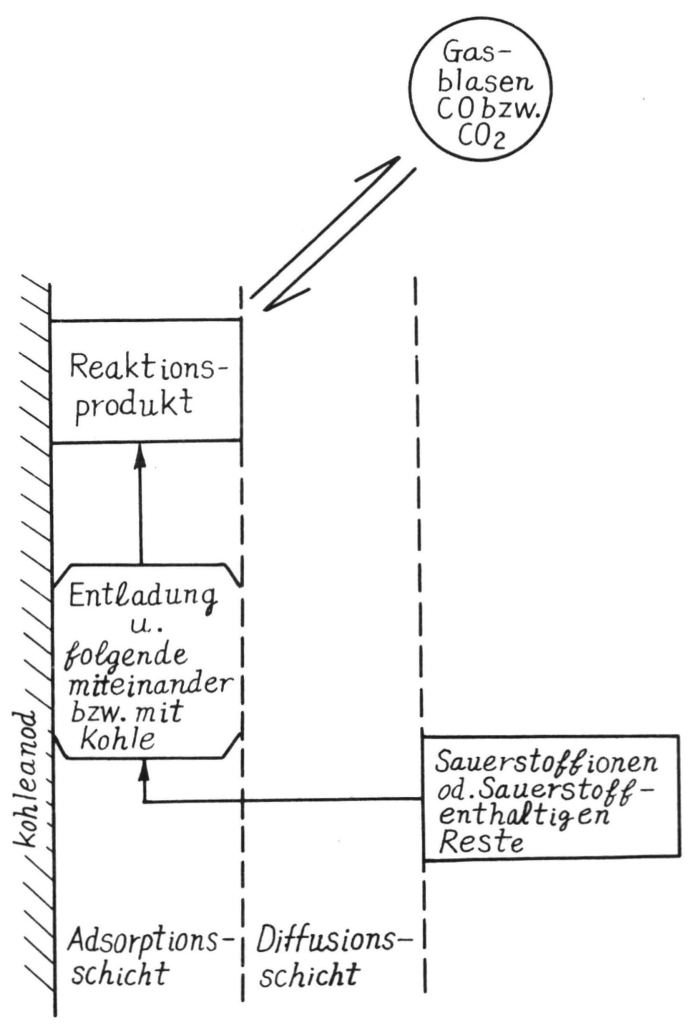

Abb. 1

1) die Heranführung der Sauerstoffionen bzw. der Sauerstoffhaltigen Reste. Würden die Fluorionen sich dabei am Reaktionsprozess beteiligen, brauchte man wegen ihrer höheren Konzentration ihren Transport trotzdem nicht zu berücksichtigen.

2) ihre Entladung und folgende Reaktionen miteinander bzw. mit der Kohleanode in ihrem an der Elektrodenoberfläche adsorbierten Zustand.

3) den Abtransport der Reaktionsprodukte, oder bei ganz kleinen Stromdichten (vielleicht höchstens von der Grössenordnung von Mikroampere pro $\mathrm{cm}^{2}$ ) nur durch Abdiffusion in den Elektrolyt, bei größeren Stromdichten parallel dazu auch in Form 
von Gasblasen erfolgt. Dafür haben wir angenommen, daß die Abdiffusion unter den Bedingungen unserer Elektrolyse als transportbestimmende Erscheinung außer Berücksichtigung bleibt.

In der ersten Näherung setzt sich die beobachtete Gessamtpolarisation $\eta$ aus der Diffusionspolarisation $\eta_{\mathrm{d}}$, der Reaktionspolarisation $\eta_{\mathrm{r}}$, der Durchtrittspolarisation $\eta_{\mathrm{D}}$ und der Widerstandspolarisation $\eta_{\mathrm{w}}$ additive zusammen, um die Ermitterung für die Elektrodenmechanismen zu vereinfachern. Bei dieser Elektrolyse ist die Durchtrittspolarisation wegen der genügend hohen Temperatur vielleicht vernachlässigbar, und für die Widerstandspolarisation, die aus der Adsorption der entwickelten Anodengase-nämlich Aufbau einer Gas-Deckschicht berüht, haben wir angenommen, daß sie zwar sicher eine Funktion der Stromdichte ist, aber schon bald nach dem Einschalten des Stroms einen konstanten Wert erreicht.

\section{Diffusion spol arisation}

Für den Transport zur Elektrode mü $\beta$ man in der Regel gleichzeitig Rücksicht auf die Diffusion, Konvektion und Migration nehmen. Bei der Elektrolyse geschmolzener Salze aber ist die Migration wegen der hohen Konzentration der Trägerionen jedenfalls zu vernachlässigen.

Bei dieser Elektrolyse tritt die Konvektion ohne Wartezeit durch die aus Elektrode entwickelten Gasblasen auf der Anode auf, kann man daher in der ersten Näherung bej vorgegebener Stromdichte eine konstante Nernstsche Diffusionsschicht voraussetzen, um die Diffusionsüberspannung als Funktion der Zeit zu bekommen.

Zur genauen Ermittlung dieser Prozesse muß die Löslichkeit von $\mathrm{CO}, \mathrm{CO}_{2}$ bzw. $\mathrm{O}_{2}$ in dieser Schmelze untersucht werden. Wir konnten leider keine Werte finden, aber sie sind vielleicht niedriger wie in wässriger Lösung.
(1) Der Fall, daß die Nachlieferung der potentialbestimmenden Komponente im Elektrolytraum nur durch die Diffusion von $\mathrm{O}^{2-}$ erfolgt 4)5) und die Entladung sehr schnell geht, nämlich

$$
\begin{aligned}
& \mathrm{O}^{2-}+\ldots \rightarrow \rightarrow \mathrm{O}^{2-}- \\
& \quad \text { (Diffusion) , (Entlandung) }
\end{aligned} \mathrm{O}_{\mathrm{ads}}+2 \mathrm{e}
$$

Für die Konzentration von $\mathrm{O}^{2-}$ gilt die bekannte Differentialgleichung

$$
\frac{\partial C_{\mathrm{o} 2-}}{\partial t}=D \frac{\partial^{2} C_{\mathrm{o}} 2-}{\partial x^{2}}
$$

mit der Anfangsbedingung

$$
C_{\mathrm{o}^{2-}}(x, o)=C_{\mathrm{o}^{2-}}^{*}
$$

Wenn der Abstand bei der Elektroden gross genug ist, dass sie einander nicht beeinflußen, kann man die Kathode mathematisch als unendlich weit von der Anode entfernt ansetzen. Wenn die Lage der Anode bei $\mathrm{x}=\mathrm{O}$ angenommen wird (Abb. 2), gelten dazu folgende Randbedingungen:

$$
\begin{aligned}
& D\left(\partial C_{\mathrm{o}^{2-}} / \partial x\right)_{x=0}=i \gamma \\
& C_{\mathrm{o} 2-}(\delta, t)=C_{\mathrm{o}^{2-}}^{*}
\end{aligned}
$$

wobei

$C_{0} 2-$ : Konzentration von $\mathrm{O}^{2-}\left(\mathrm{Mol} / \mathrm{cm}^{3}\right)$

$C_{02-}^{*}$ : Losungskonzentration von $\mathrm{O}^{2-}$ $\left(\mathrm{Mol} / \mathrm{cm}^{3}\right)$

$D$ : Diffusionskonstante von $\mathrm{O}^{2-}$ $\left(\mathrm{cm}^{2} / \mathrm{sec}\right)$

$i$ : Stromdichte an der Anode (Amp/ $\mathrm{cm}^{2}$ )

$\gamma: 1 / 2 \mathrm{Fr}$ (Fr: Faradaysche Konstante (abs.Coul. /gr. $\ddot{\text { Aquiv.)) }}$

$\delta \quad:$ Dicke der Diffusionsschicht (cm)

$x$ : Entfernung von der Anode (cm)

$t$ : Zeit nach dem Einschalten (sec)

Die Differentialgleichung (1-1) mit den in $\mathrm{Gl}(1-3)$ gegebenen Randbedingungen durch 


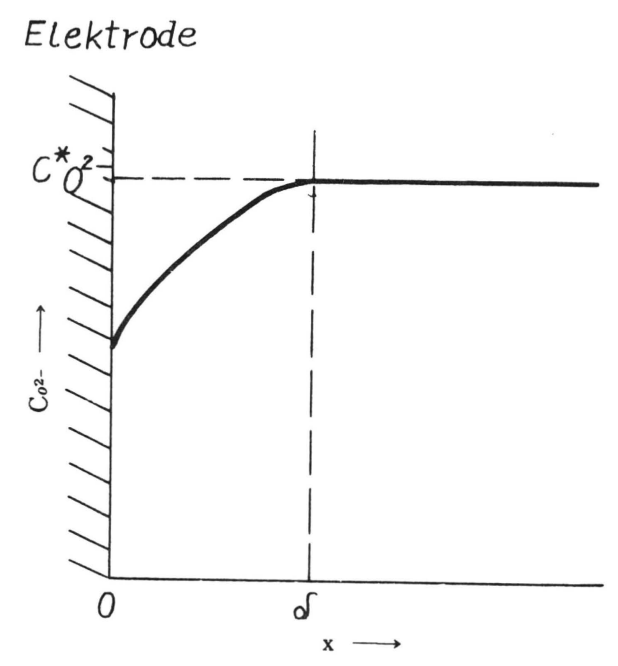

Abb. 2

die Anwendung der Laplace-Transformation ganz einfach gelöst werden kann, obwohl die allgemeine Lösung als mathematisch wesentlich komplizierter Fall schon von Rosebruch und Miller ${ }^{9}$ ) in Form einer Fourierschen Reihe erhalten wurde:

$$
\begin{aligned}
& C_{\mathrm{o}^{2-}}=C_{\mathrm{o}^{2-}}^{*}-\frac{i \gamma \delta}{D} \\
& +\frac{8 i \gamma \delta}{D_{\pi^{2}}} \sum_{n=o}^{\infty} \frac{\exp \left\{-\left(\frac{\pi(1+2 n)}{2 \delta}\right)^{2} D t\right\}}{(1+2 n)^{2}}
\end{aligned}
$$

Hieraus bekommen wir die Diffusionspolarisation für Prozeß (I-i)

$$
\eta_{d}=-\frac{R T}{2 F_{r}} \ln \frac{C_{\mathrm{o}^{2-}}(o, t)}{C_{\mathrm{o}^{2-}}^{*}}
$$

Nimmt man besonders an, daß $\mathrm{Al}_{2} \mathrm{O}_{3}$ in der Schmelze vollkommen $\mathrm{zu} \mathrm{Al}^{3+}$ und $\mathrm{O}^{2-}$ dissoziert ist, so gilt notwendigerweise

$$
C_{\mathrm{O}^{2-}}^{*}=3 C^{*}
$$

wenn $C$ die Lösungskonzentration an $\mathrm{Al}_{2} \mathrm{O}_{3}$ $\left(\mathrm{Mol} / \mathrm{cm}^{3}\right)$ im Elektrolyt ist. Schließlich ergibt sich die gesuchte Diffusionspolarisation

$$
\begin{aligned}
\eta_{d} & =-\frac{R T}{2 F_{r}} \ln \left[1-\frac{i \delta}{6 D C^{*} F_{r}}\right. \\
& +\frac{4 i \delta}{3 D \pi^{2} C^{*} F_{r}} \sum_{n=o}^{\infty} \frac{\exp \left\{-\left(\frac{\pi(1+2 n)}{2 \delta}\right)^{2} D t\right\}}{(1+2 n)^{2}}
\end{aligned}
$$

Bei der praktischen Anwendung dieser Gleichung ist der Ausdruck der Konzentration C* etwas problematisch, weil die Dichte der Schmelze, in der unsere Messungdurchgeführt wurde, nicht genau bekannt ist. Wir haben aber die Konzentration $\left(\mathrm{Mol} / \mathrm{cm}^{3}\right.$ ) näherungsweise unter der Annahme ausgerechnet, daß die Dichte der Schmelze durch ihre Hauptiuestandteil $\mathrm{Na}_{3} \mathrm{AlF}_{6}$ (ca.70 Gew.\%) bestimmt ist. Folglich haben wir erhalten

$$
C^{*}=6,2 \cdot 10^{-4} \mathrm{in}_{\mathrm{i}} \mathrm{ol} / \mathrm{cm}^{3}
$$

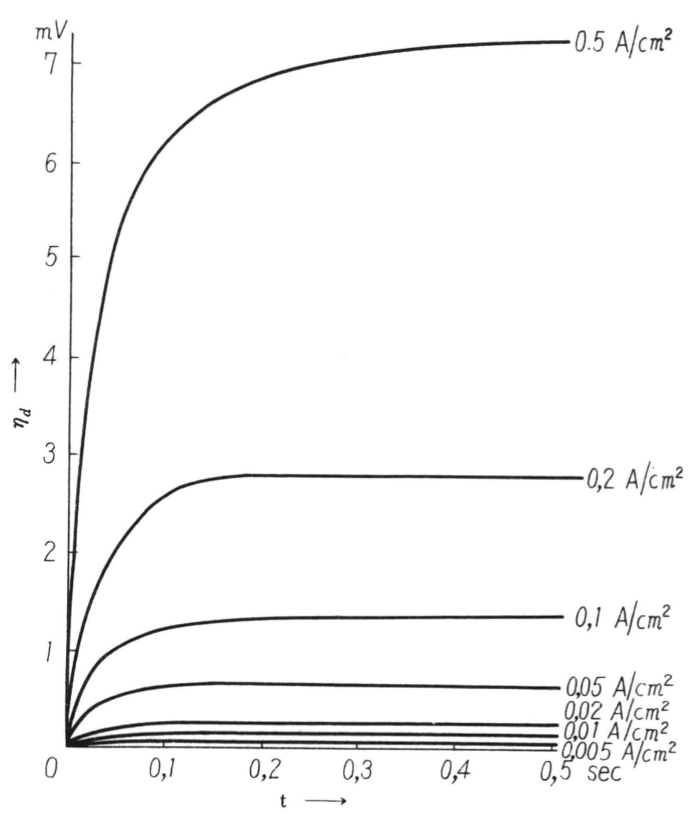

Abb. 3

Abb. 3 ist nun als ein mögliches Beispiel

$$
\left(\delta=10^{-3} \mathrm{~cm}, \mathrm{D}=10^{-5} \mathrm{~cm}^{2} / \mathrm{sec}\right)
$$


aus der numerischen Rechnung hervorgegangen, um die Eigenschaften der Funktion deutlich zu zeigen.

(2) Der Fall, dass $\mathrm{Al}_{2} \mathrm{O}_{3}$ im Elektrolyt nicht dissoziert (oder in der Form eines Restes mit 3 Sauers toffatomen enthalten) ist, die Nachlieferung von Sauerstoff an die Elektrodenoberfläche nur durch die Diffusion von $\mathrm{Al}_{2} \mathrm{O}_{3}$ erfolgt und anschließend eine Reaktion mit dem bereits entladenen Fluor stattfindet 4)5)10)11)12)

Dabei braucht man wegen der hohen Konzentration der Fluorionen ihre Diffusion nach oder von der Elektrode nicht zu berücksichtigen, und wir nehmen an, daß diese Reaktion von atomarem Fluor mit $\mathrm{Al}_{2} \mathrm{O}_{3}$ sehr schnell geht.

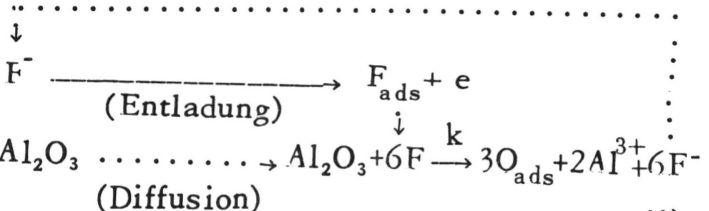

(Diffusion)

An der Elektrodenoberfläche gilt

$$
\frac{D C F}{d t}=2 i \gamma-6 k C_{F}^{6} \cdot C_{A l_{2} O_{3}}(o, t)
$$

wenn $\mathrm{C}_{\mathrm{Al}_{2} \mathrm{O}_{3}}$ dië Konzentration von $\mathrm{Al}_{2} \mathrm{O}_{3}$ $\left(\mathrm{Mol} / \mathrm{cm}^{2}\right) \mathrm{C}_{\mathrm{F}}$ die Konzentration von Fluor an der Elektrode $\left(\mathrm{Mol} / \mathrm{cm}^{2}\right)$ und die $k \mathrm{Ge}$ schwindigkeitskonstante ist. Im Elektrolyt gilt die Diffusionsgleichung

$$
\frac{\partial C_{\mathrm{Al}_{2} \mathrm{O}_{3}}}{\partial t}=D \frac{\partial^{2} C_{\mathrm{Al}_{2} \mathrm{O}_{3}}}{\partial x^{2}}
$$

mit den Randbedingungen

$$
\begin{aligned}
& D\left(\frac{\partial C \mathrm{Al}_{2} \mathrm{O}_{3}}{\partial x}\right)_{x=0}=k C_{\mathrm{F}}^{6} \cdot C_{\mathrm{Al}_{2} \mathrm{O}_{3}}(o, t) \\
& C_{\mathrm{Al}_{2} \mathrm{O}_{3}}(\delta, t)=C^{*}
\end{aligned}
$$

und mit den Anfangsbedingungen

$$
\begin{aligned}
& C_{\mathrm{Al}_{2} \mathrm{O}_{3}}(x, o)=C^{*} \\
& C_{\mathrm{F}(t=0)}=0
\end{aligned}
$$

wenn $\mathrm{D}$ diesmal die Diffusionskonstante von $\mathrm{Al}_{2} \mathrm{O}_{3}\left(\mathrm{~cm}^{2} / \mathrm{sec}\right)$ ist. Es ist wegen der besonders ausgeprägten Nichtlinearität sicher unmöglich, obiges Differntialgleichungssystem unmittelbar zu lösen. Durch die Annahme, daß $\mathrm{K}$ außerordentlich groß ist, läßt sich die mathematischen Behandlung wesentlich verein fachen, nä dich

$$
\lim _{k \rightarrow \infty} C_{\mathrm{F}}=0 \text { und } \lim _{k \rightarrow \infty} \frac{d C_{\mathrm{F}}}{d t}=0
$$

Es gilt also aus $\mathrm{Gl}\left(2^{-/} / 1\right)$ an der Elektrode

$$
k C_{1}^{6} \quad C_{\mathrm{Al}_{2} \mathrm{O}_{3}}(o, t)=2 i \gamma
$$

und folglich können wir aus $\mathrm{Gl}(2-3)$ als lineare Randbedingung von $\mathrm{Gl}(2-2)$

$$
D\left(\frac{\partial C_{\mathrm{Al}_{2} \mathrm{O}_{3}}}{\partial x}\right)_{x=0}=\frac{i y}{3}
$$

erhalten. In gleicher Weise wird die gesuchte Diffusionspolarisation für den Prozess (I-ii) durch den Ausdruck

$$
\begin{aligned}
\eta_{d} & =-\frac{R T}{6 F_{r}} \ln \left[1-\frac{i \delta}{6 D C^{*} F_{r}}\right. \\
& \left.+\frac{4 i \delta}{3 D^{2} C^{*} F_{r}} \sum_{n=0}^{\infty} \frac{\exp \left\{-\left(\frac{\pi(1+2 n)}{2 \delta}\right)^{2} D_{t}\right\}}{(1+2 n)^{2}}-\right]
\end{aligned}
$$

dargestellt.

(3) Der Fall, dass Sauerstoff als Rest mit einem Sauerstoffatom $\mathrm{MO}^{\mathrm{n}} \stackrel{ \pm}{,}$ z.B. $\mathrm{Na}_{2} \mathrm{O}^{1) 13)}$, $[\mathrm{AlO}]^{+6) 7)},\left[\mathrm{AlOF}_{2}^{-}\right]^{14)},\left[\mathrm{AlOF}_{4}\right]^{3-}$ usw., transportiert wird, also ähnlich wie bei Prozess (I-i) 


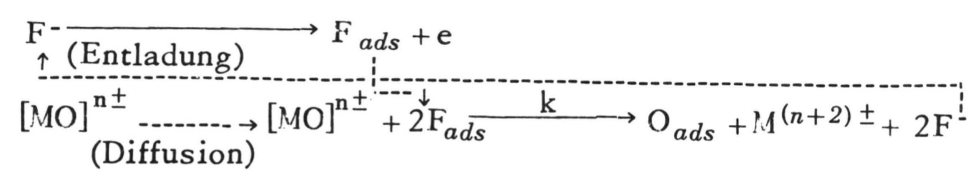

Selbst wenn ein Rest wie z.B. $\left[\mathrm{AlOF}_{2}\right]$ Sauerstoff abgibt und vorübergehend das in der Schmelze seltene und damit diffusionsbestimmende Ion $\left[\mathrm{AlF}_{2}\right]^{+}$entstünde, nehmen wir an, $\mathrm{da} \beta$ diese Ion fast sofolt zersetzt wird ( in unserem Reispiel $\mathrm{Al}^{3^{+}}, \mathrm{F}^{-}$) sodaß sich unsere Differentialgleichung in einer ähnlichen Näherung wie vorher darstellen läßt.

$$
\frac{\partial C_{\text {MOn士 }}}{\partial t}=D \frac{\partial^{2} C_{M O n \pm}}{\partial x^{2}}
$$

mit den Anfangs- und Randbesingungen

$$
C_{\mathrm{MO}^{n \pm}}(x, o)=C_{\mathrm{MO}{ }^{ \pm}}^{*} \quad D\left(\frac{\partial C_{\mathrm{MO}^{n \pm}}}{\partial x}\right)_{x=0}=i \gamma \quad C_{\mathrm{MO}^{n \pm}}(\delta, t)=C^{*}{ }_{\mathrm{MO}^{n \pm}}
$$

wobei D die Diffusionskonstante von $[M O]^{\mathrm{n} \pm}$ ist. Hieraus ergibt sich sofolt die Diffusionspolarisation

$$
\eta_{d}=-\frac{R T}{2 F_{r}} \ln \left[1-\frac{i \delta}{6 D F_{r} C^{*}}+\frac{4 i \delta}{3 D \pi^{2} C^{*} F_{r}} \sum_{n=0}^{\infty} \frac{\exp \left\{-\left(\frac{\pi(1+2 n)}{2 \delta}\right)^{2} D t\right\}}{(1+2 n)^{2}}\right.
$$

Wenn man die Abdiffusion von $M^{(n \pm 2) \pm}$ berücksichtigen $m u ß$, gilt dafür die Differntialgleichung (3-1)/(3-2) mit dem Zusatz

$$
-\frac{\partial C^{\prime}}{\partial t}=D \frac{\partial^{2} C^{\prime}}{\partial x^{2}}
$$

mit den Anfangs- und Randbedingungen

$$
C^{\prime}(x, o)=0 \quad D\left(\frac{\partial C^{\prime}}{\partial x}\right)_{x=0}=-i \gamma \quad C^{\prime}(\delta, t)=0
$$

wenn

$$
\begin{aligned}
& D^{\prime}: \text { Diffusionskonstante von } \mathrm{M}^{(\mathrm{n} \pm 2) \pm}\left(\mathrm{cm}^{2} / \mathrm{sec}\right) \\
& C^{\prime}: \text { Konzentration von } \mathrm{M}^{(\mathrm{n} \pm 2) \pm}\left(\mathrm{Mol} / \mathrm{cm}^{3}\right)
\end{aligned}
$$

Auf Grund der Elektrochemie ergibt sich aus den Lösungen von $\mathrm{Gl}(3-1)$ und $\mathrm{Gl}(3-4)$ 


$$
\eta_{d}=-\frac{R T}{2 F_{r}} \ln \left[\frac{3 C^{*}-\frac{i \delta}{2 D F_{r}}+\frac{4 i \delta}{D \pi^{2} F_{r}} \sum_{n=0}^{\infty} \frac{\exp \left\{-\left(\frac{\pi(1+2 n)}{2 \delta}\right)^{2} D_{t}\right\}}{(1+2 n)^{2}}}{\frac{i \delta}{2 D F_{r}}-\frac{4 i \delta}{D \pi^{2} F_{r}} \sum_{n=0}^{\infty} \frac{\exp \left\{-\left(\frac{\pi(1+2 n)}{2 \delta}\right)^{2} D_{t}\right\}}{(1+2 n)^{2}}-}\right]
$$

wenn $\mathrm{D}=\mathrm{D}^{\prime}$ ist.

(4) Der Fall, daB der Elektrodenvorgang durch einen Rest mit zwei Sauerstoffatomen $\left[\mathrm{MO}_{2}\right]^{\mathrm{n}-}$, z.B. $\left[\mathrm{AlO}_{2}\right]_{;}^{-}\left[\mathrm{AlO}_{2} \mathrm{~F}_{2}\right]^{3-5) 7)}$ usw., bestimmt wird, also

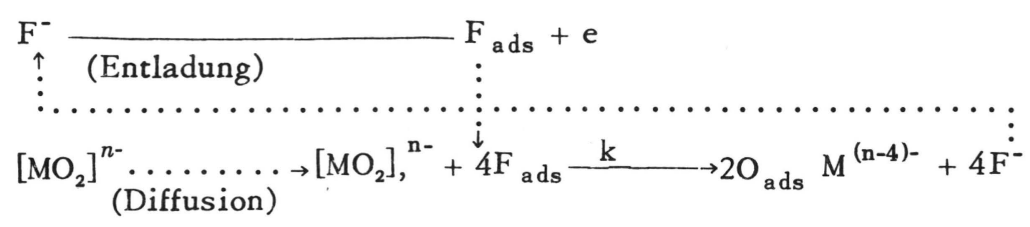

Dabei ergibt sich die Diffusionspolarisation ähnlich wie bei der vorherigen Behandlung

$$
\eta_{d}=-\frac{R T}{4 F_{r}} \ln \left[1-\frac{i \delta}{6 D C^{*} F_{r}}+\frac{4 i \delta}{3 D \pi^{2} C^{*} F_{r}} \sum_{n=0}^{\infty} \frac{\exp \left\{-\left(\frac{\pi(1+2 n)}{2 \delta}\right)^{2} D t\right\}}{(1+2 n)^{2}}\right]
$$

unter der Annahme, daß $\mathrm{M}^{(\mathrm{n}-4)-}$ sofort in die einfachen Ionen zerfällt, deren Konzentration in der Schmelze groß ist, selbst wenn $M^{(n-4)-}$ das diffusionsbestimmende Ion wäre.

\section{Reaktion spolarisation}

Beim Problem der Reaktionspolarisation können wir die Berechnung sehr vereinfachen durch die Annahme, daß die Reaktion zwischen Sauerstoff und Kohle wegen des aktiverten Zustandes des Sauerstoffs gleich nach der Entladung sehr schnell erfolgt. Die Tatsache, daß Sauerstoff im Anodengas nicht nachweisbar ist, bestätigt unsere Annahme und beweist gleichzeitig, da $\beta$ der Sauerstoff keine Gelegenheit hat, zu rekombinieren.

(5) Der Fall, daß der entladene Sauerstoff unter Bildung von CO mit der Kohl eanode reagiert und das so gebildete und noch adsorbierte $\mathrm{CO}$ über das Gleichgewicht mit freiem gasförmigen $\mathrm{CO}$ desorbiert wird mit dem Anodengas abgeht.

$$
\mathrm{O}_{\mathrm{ads}}+\mathrm{C} \stackrel{k_{\mathrm{co}}}{\mathrm{CO}} \underset{\mathrm{ads}}{\stackrel{k_{c o}}{\underset{k_{c o}^{m}}{\longrightarrow}}} \mathrm{CO}_{\mathrm{gas}}
$$

Für den Prozeß(II-i) gilt an der Anode die Differntialgleichung

$$
\frac{d C_{\mathrm{o}}}{d t}=i y-k_{\mathrm{co}} C_{o} \quad(5-1) \quad \frac{d C_{\mathrm{co}}}{d t}=k_{\mathrm{co}} C_{\mathrm{o}}-k_{\mathrm{co}} C_{\mathrm{co}}+\lambda_{\mathrm{co}}
$$


mit den Anfangsbedingungen

$$
C_{\mathrm{o}}(o)=0 \quad C_{c o}(o)=0
$$

Wenn jede Konzentration in $\mathrm{Mol} / \mathrm{cm}^{2}$ gemessen wird, und

$$
\lambda_{\mathrm{co}}=k_{\mathrm{co}} P_{\mathrm{co}}
$$

wobei P co der Druck der CO-Gasblasen ist. Offensichtlich darf man $\mathrm{Pco}$ als konstant betrachten, weil Pco der Druck der COGasblasen (ca 1 atm.) ist. Mittels der Laplace-Transformation erhalten wir ganz einfach

$$
\begin{aligned}
C_{\mathrm{o}} & =\frac{i y_{c o}}{k_{c o}}\left\{1-\exp \left(-k_{\mathrm{co}} t\right)\right\} \\
C_{\mathrm{co}} & =\frac{i \gamma}{k_{\mathrm{co}}}+\frac{\lambda_{\mathrm{co}}}{k_{\mathrm{co}}}\left\{1-\exp \left(-k_{\mathrm{co}} t\right)\right\} \\
+ & \frac{i \gamma\left\{k_{\mathrm{co}}^{\prime} \exp \left(-k_{\mathrm{co}} t\right)-k_{\mathrm{co}} \exp \left(-k_{\mathrm{co}}^{\prime} t\right)\right\}}{k_{\mathrm{co}}^{\prime}\left(k_{\mathrm{co}}-k_{\mathrm{co}}^{\prime}\right)}
\end{aligned}
$$

Nach der Annahme $k_{\text {co }} \rightarrow \infty$ ergibt sich sofort

$$
\begin{aligned}
& C_{\mathrm{o}}=0 \\
& C_{\mathrm{co}}=\frac{i \gamma+\lambda_{\mathrm{co}}}{k_{\mathrm{co}}^{\prime}}\left\{1-\exp \left(-k_{\mathrm{co}}^{\prime} t\right)\right\}
\end{aligned}
$$

Hieraus ergibt sich die Reaktionspolarisation

$$
\eta_{r}=\frac{k T}{2 F_{r}} \ln \frac{\left(i \gamma \neq \lambda_{\mathrm{co}}\right)\left\{1-\exp \left(-k_{\mathrm{co}}^{\prime} t\right)\right\}}{3 k^{\prime}{ }_{\mathrm{co}} C^{*}}(5-7)
$$

(6) Der Fall, dass $\mathrm{CO}_{2}$ statt $\mathrm{CO}_{2}$ (beim Proze $\beta$ (11-i) gebildet wird, nämlich

$2 \mathrm{O}_{\mathrm{ads}}+\mathrm{C} \frac{k \mathrm{co}_{2}}{\mathrm{CO}_{2} \text { ads }} \underset{k^{n}{ }_{\mathrm{co}_{2}}^{\prime}}{\stackrel{k_{\mathrm{Co}_{2}}}{\leftrightarrows}} \mathrm{CO}_{2}$ gas

Hier gelten, ähnlich wie vorher, folgende Differentialgleichungen

$$
\begin{aligned}
& \frac{d C_{\mathrm{o}}}{d t}=i \gamma-2 k_{\mathrm{co}_{2}} C_{\mathrm{o}}^{2} \\
& \frac{d C_{\mathrm{co}_{2}}}{d t}=k_{\mathrm{co}} C_{\mathrm{o}}^{2}-k_{\mathrm{Co}_{2}} C_{\mathrm{co}_{2}}+\lambda_{\mathrm{co}_{2}}
\end{aligned}
$$

mit den Anfangsbedingungen

$$
\begin{aligned}
& C_{\mathrm{o}}(o)=0 \\
& C_{\mathrm{Co}_{2}}(o)=0
\end{aligned}
$$

wenn ähnlich zu Fall (5)

$$
\lambda_{\mathrm{co}_{2}}=k_{\mathrm{CO}_{2}} p_{\mathrm{co}_{2}}
$$

(Konstante)

ist.

Zuerst kann man die RiccatischeDifferentialgleichung (6-1) ohne Schwierigkeit lösen, nähmlich

$$
\begin{aligned}
C_{\mathrm{o}}= & \frac{\left(4 k_{\mathrm{co}_{2}} i \gamma-1\right)\{1-\exp }{\left(4 k_{\mathrm{co}_{2}} i \gamma-1\right)\left\{1-\exp \left(\frac{-t}{2 \sqrt{2 k_{\mathrm{co}_{2}} i \gamma}}\right)\right\}} \\
& \left.\left.\times \sqrt{\frac{i \gamma}{2 k_{\mathrm{co}_{2}} i \gamma}}\right)\right\}-1
\end{aligned}
$$

Hieraus ergibt sich sofort für $k_{\mathrm{co}_{2}} \rightarrow \infty$

$$
\begin{aligned}
& C_{\mathrm{o}}=0 \\
& k_{\mathrm{co}_{2}} C_{\mathrm{o}}^{2}=\frac{i \gamma}{2}
\end{aligned}
$$

folglich ist Gl (6-2)

$$
\frac{d C_{\mathrm{co}_{2}}}{d t}=\frac{i \gamma}{2}-k_{\mathrm{CO}_{2}}^{\prime} C_{\mathrm{co}_{2}}+\lambda_{\mathrm{co}_{2}}
$$

Die Lösung dieser Gleichung wird ganz einfach durch den Ausdruck

$$
C_{\mathrm{co}_{2}}=\frac{i \gamma+2 \lambda \mathrm{co}_{2}}{2 k_{\mathrm{co}}^{\prime}}\left\{1-\exp \left(-k_{\mathrm{Co}_{2}}^{\prime} t\right)\right\}
$$


gegeben. Damit ist die gesuchte Reaktionspolarisation

$$
\eta_{r}=\frac{R T}{4 F_{r}} \ln \frac{\left(i \gamma+2 \lambda_{\mathrm{co}_{2}}\right)\left\{1-\exp \left(-k^{\prime} \mathrm{co}_{2} t\right)\right\}}{18 k^{\prime} \mathrm{co}_{2} C^{* 2}}
$$

ähnlich zu Gl (5-7).

(7) Beim Fall, da $\beta$ die Reaktionen

$$
\mathrm{O}_{\mathrm{ads}}+\mathrm{C} \stackrel{\mathrm{k}_{\mathrm{co}}}{\longrightarrow} \mathrm{CO}_{\mathrm{ads}} \stackrel{\mathrm{k}_{\mathrm{co}}}{\underset{\mathrm{k}_{\mathrm{co}}^{n}}{\longrightarrow}} \mathrm{CO}_{\mathrm{ads}}
$$

$$
20_{\mathrm{ads}}+\mathrm{C}-\stackrel{\mathrm{k}_{\mathrm{co}}}{\longrightarrow} \mathrm{CO}_{2} \text { ads } \underset{\mathrm{k}_{\mathrm{CO}_{2}}^{\prime}}{\stackrel{\mathrm{k}_{\mathrm{co}}}{\longrightarrow}} \mathrm{CO}_{2} \text { gas }
$$

gleichzeitig an der Anodenoberfläche stattfinden, genügen $C_{\mathrm{o}}, C_{\text {co }}$ und $C_{\mathrm{Co}_{2}}$ für die Differntialgleichungen

$$
\begin{aligned}
& \frac{d C_{\mathrm{o}}}{d t}=i \gamma-k_{\mathrm{co}} C_{\mathrm{o}}-2 k_{\mathrm{co}_{2}} C_{\mathrm{o}}^{2} \\
& \frac{d C_{\mathrm{co}}}{d t}=k_{\mathrm{co}} C_{\mathrm{o}}-k_{\mathrm{co}}^{\prime} C_{\mathrm{co}}+\lambda_{\mathrm{co}} \\
& \frac{d C_{\mathrm{co}_{2}}}{d t}=k_{\mathrm{co}_{2}} C_{\mathrm{o}}^{2}-k_{\mathrm{co}_{2}} C_{\mathrm{co}_{2}}+\lambda_{\mathrm{co}_{2}}
\end{aligned}
$$

mit den Anfangsbedingungen

$$
\begin{aligned}
& C_{\mathrm{o}}(o)=0 \\
& C_{\mathrm{co}}(o)=0 \\
& C_{\mathrm{Co}_{2}}(o)=0
\end{aligned}
$$

in deren

$$
\lambda_{\mathrm{co}}=k_{\mathrm{co}}^{n} p_{\mathrm{co}} \text { und } \lambda_{\mathrm{co}_{2}}=k_{\mathrm{co}_{2}}^{n} p_{\mathrm{co}_{2}}
$$

sind, wenn $P_{\text {co }}$ und $P_{\text {-co }}$ die Pertialdrucke von $\mathrm{CO}$ und $\mathrm{CO}_{2}$ in den Gasblasen sind. Dabei kann man auch $\lambda_{\text {co }}$ und $\lambda_{\mathrm{Co}_{2}}$ als Konstant betrachten, wenn das Verhältnis- von $\mathrm{CO}$ und $\mathrm{CO}_{2}$ in den Gasblasen immer Konstant ist.

Man erhält die gesuchte Lösung der Riccatischen Differential-gleichung (7-1) wie üblich:

$$
\begin{aligned}
& C_{\mathrm{o}}= \\
& \frac{U\left\{2 k_{\mathrm{co}_{2}} U\left(k_{\mathrm{co}}+4 k_{\mathrm{co}_{2}} U\right)-1\right\}\left\{1-\exp \left(\frac{-t}{k \mathrm{co}+4 k_{\mathrm{co}_{2}} U}\right)\right\}}{2 k_{\mathrm{co}_{2}} U\left(k_{\mathrm{co}}+4 k_{\mathrm{co}_{2}} U\right)\left\{1-\exp \left(-\frac{-t}{k_{\mathrm{co}}+4 k_{\mathrm{co}_{2}} U}\right)\right\}-1}
\end{aligned}
$$

wenn die stationäre Lösung von $C_{o}$

$$
U=\frac{-k_{\mathrm{co}}+\sqrt{k_{\mathrm{co}}^{2}+8 k_{\mathrm{co}_{2}} i \gamma}}{4 k_{\mathrm{co}_{2}}}
$$

ist.

Hieraus ergibt sich weiter für $k_{\mathrm{co}}, k_{\mathrm{co}_{2}} \rightarrow \infty$

$$
\begin{aligned}
& C_{\mathrm{o}}=0 \\
& k_{\mathrm{co}} C_{\mathrm{o}}=i \gamma \\
& k_{\mathrm{co}_{2}} C_{\mathrm{o}}^{2}=0
\end{aligned}
$$

Damit lassen sich $\mathrm{Gl}(7-2)$ und $\mathrm{Gl}(7-3)$ in der Gestalt

$$
\frac{d C_{\mathrm{co}}}{d t}=i \gamma+\lambda_{\mathrm{co}}-k_{\mathrm{co}}^{\prime} C_{\mathrm{co}}
$$

und

$$
\frac{d C_{\mathrm{CO}_{2}}}{d t}=\lambda \lambda_{\mathrm{CO}_{2}}-k \mathrm{CO}_{2} C_{\mathrm{co}_{2}}
$$

dargestellen

Als Lösung dieser Differentialgleichungen ergeben sich einfach die Gleichung

$$
\begin{aligned}
& C_{\mathrm{co}}=\frac{i y+\lambda_{\mathrm{co}}}{k_{\mathrm{co}}^{\prime}}\left\{1-\exp \left(-k_{\mathrm{co}}^{\prime} t\right)\right\} \\
& C_{\mathrm{co}_{2}}=\frac{\lambda_{\mathrm{co}}}{k{ }_{\mathrm{CO}_{2}}}\left\{1-\exp \left(-k_{\mathrm{Co}_{2}}^{\prime} t\right)\right\}
\end{aligned}
$$


Schließlich erhalten wir

$$
\eta_{r}=\frac{R T}{6 F_{r}}\left[\ln \frac{\left(i \gamma+\lambda_{\mathrm{co}}\right)\left\{1-\exp \left(-k_{\mathrm{co}}^{\prime} t\right)\right\}}{3 k_{\mathrm{co}}^{\prime} C^{*}}+\ln \frac{\lambda_{\mathrm{co}_{2}}\left\{1-\exp \left(-k_{\mathrm{co}_{2}}^{\prime} t\right)\right\}}{9 k_{\mathrm{co}_{2}}^{\prime} C^{* 2}}\right]
$$

(8) Beim Fall, daß die stufenweise Reaktion des Sauerstoffs

$$
\begin{aligned}
& \mathrm{O}_{\mathrm{ads}}+\mathrm{C} \frac{k_{\mathrm{co}}}{\mathrm{CO}} \mathrm{ads} \frac{k_{\mathrm{co}}^{\prime}}{k_{\mathrm{co}}^{m}} \mathrm{CO}_{\mathrm{gas}}
\end{aligned}
$$

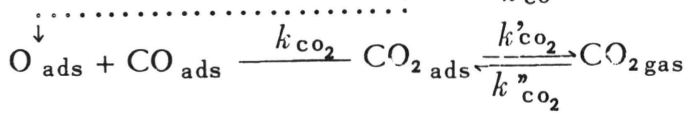

an der Electrodenoberfläche $\mathrm{zu} \mathrm{CO}_{2}$ führt, gelten die nichtlinearen Differentialgleichungen

$$
\begin{aligned}
& \frac{d C_{\mathrm{o}}}{d t}=i \gamma-k_{\mathrm{co}} C_{\mathrm{o}}-k_{\mathrm{co}_{2}} C_{\mathrm{co}} C_{\mathrm{o}} \\
& \frac{d C_{\mathrm{co}}}{d t}=k_{\mathrm{co}} C_{\mathrm{o}}-k_{\mathrm{co}_{2}} C_{\mathrm{co}} C_{\mathrm{o}}-k_{\mathrm{co}}^{\prime} C_{\mathrm{co}}+\lambda_{\mathrm{co}} \\
& \frac{d C_{\mathrm{co}_{2}}}{d t}=k_{\mathrm{co}_{2}} C_{\mathrm{co}} C_{\mathrm{o}}-k_{\mathrm{co}_{2}}^{\prime} C_{\mathrm{co}_{2}}+\lambda_{\mathrm{co}_{2}}
\end{aligned}
$$

mit den Anfangsbedingungen

$$
C_{\mathrm{o}}(o)=0 \quad C_{\mathrm{co}}(o)=0 \quad C_{\mathrm{co}_{2}}(o)=0
$$

Es ist ziemlich kompliziert dieses System von nichtlinearen Differentialgleichungen genau zu berechnen Erzielt man aber zuerst eine Näherungslösung für die Sauerstoffkonzentration $C_{0}$, die bei hsehr großen Werten von $k$ co und $k \mathrm{co}_{2}$ sicher Null wird, dann ergibt sich $C_{\text {co und }}$ $\mathrm{C}_{\mathrm{co}_{2}}$ ganz einfach. Daher haben wir nach der in Anhang 2. beschriebenen Behandlung erhalten:

$$
\begin{aligned}
& C_{\mathrm{o}}\left(\text { bei } k_{\mathrm{co}}, k_{\mathrm{co}_{2}} \rightarrow \infty\right)=0
\end{aligned}
$$

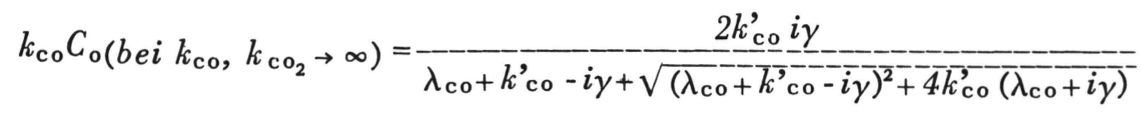

Folglich lassen sich auch $\mathrm{C}_{\mathrm{co}}$ und $\mathrm{C}_{\mathrm{co}_{2}}$, nach Anhang 2. mit den Ausdrücken

$$
\begin{aligned}
& C_{\mathrm{co}}=M\left\{1-\exp \left(-k_{\mathrm{co}}^{\prime} t\right)\right\} \\
& C_{\mathrm{co}_{2}}=N\left\{1-\exp \left(-k^{\prime}{ }_{\mathrm{co}_{2}} t\right)\right\}
\end{aligned}
$$




$$
\begin{aligned}
& M=\frac{(i \gamma)^{2}+i \gamma\left(3 k_{\mathrm{co}}^{\prime}-2 \lambda_{\mathrm{co}}\right)+\lambda_{\mathrm{co}}\left(\lambda_{\mathrm{co}}+k_{\mathrm{co}}^{\prime}\right)+\left(\lambda_{\mathrm{co}}-i \gamma\right) \sqrt{\left(\lambda_{\mathrm{co}}+k_{\mathrm{co}}^{\prime}-i \gamma\right)^{2}+4 k_{\mathrm{co}}^{\prime}\left(\lambda_{\mathrm{co}}+i \gamma\right)}}{k_{\mathrm{co}}^{\prime}\left\{\lambda_{\mathrm{co}}+k_{\mathrm{co}}^{\prime}-i \gamma+\sqrt{\left(\lambda_{\mathrm{co}}+k_{\mathrm{co}}^{\prime}-i \gamma\right)^{2}+4 k_{\mathrm{co}}^{\prime}\left(\lambda_{\mathrm{co}}+i \gamma\right)}\right\}} \\
& N=\frac{-(i \gamma)^{2}+i \gamma\left(\lambda_{\mathrm{co}}-\lambda_{\mathrm{co}_{2}}+3 k_{\mathrm{co}}^{\prime}\right)+\lambda_{\mathrm{co}_{2}}\left(\lambda_{\mathrm{co}}+k_{\mathrm{co}}^{\prime}\right)+\left(\lambda_{\mathrm{co}}+i \gamma\right) \sqrt{\left(\lambda_{\mathrm{co}}+k_{\mathrm{co}}^{\prime}-i \gamma\right)^{2}+4 k_{\mathrm{co}}^{\prime}\left(\lambda_{\mathrm{co}}+i \gamma\right)}}{k_{\mathrm{co}_{2}}^{\prime}\left\{\lambda_{\mathrm{co}}+k_{\mathrm{co}}^{\prime}-i \gamma+\sqrt{\left.\left(\lambda_{\mathrm{co}}+k_{\mathrm{co}}^{\prime}-i \gamma\right)^{2}+4 k_{\mathrm{co}}^{\prime}\left(\lambda_{\mathrm{co}}+i \gamma\right)\right\}}\right.}
\end{aligned}
$$

Hieraus ergibt sich die Reaktionspolarisation

$$
\eta_{r}=\frac{R T}{6 F_{r}}\left[\ln \frac{M\left\{1-\exp \left(-k_{\mathrm{co}}^{\prime} t\right)\right\}}{3 C^{*}}+\ln \frac{N\left\{1-\exp \left(-k_{\mathrm{co}}^{\prime} t\right)\right\}}{9 C^{* 2}}\right]
$$

Offensichtlich laßt sich unsere Näherungsmethode nicht mehr für sehr kleine Werte von $t$ $(t \rightarrow 0)$ anwenden. Aber für sehr kleines $t$ ist nicht mehr $\eta_{r}$, sondern die Doppelschichtkapazität potential-bastimmend, wie (III) beweist. Daher genügt also auch für kleine t G1 (8-10).

(9) Der Fall, daß zuerst gebildetes $\mathrm{CO}_{2}$ an Kohle sekundär re duziert wird, nämlich

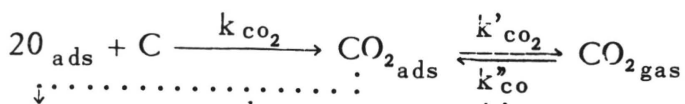

$$
\begin{aligned}
& \mathrm{CO}_{2 \mathrm{ads}}+\mathrm{C} \underset{\mathrm{k}_{\mathrm{co}}^{\prime \prime}}{\stackrel{\mathrm{k}_{\mathrm{co}}}{\rightleftharpoons}} 2 \mathrm{CO}_{\mathrm{ads}} \frac{\mathrm{k}_{\mathrm{co}}^{\prime}}{\mathrm{k}_{\mathrm{co}}^{\prime \prime}} \mathrm{CO}_{\text {gas }}
\end{aligned}
$$

Dabei gelten folgende Differentialgleichungen:

$$
\begin{aligned}
& \frac{d C_{\mathrm{o}}}{d t}=i \gamma-2 k_{\mathrm{co}_{2}} C_{\mathrm{o}}^{2} \\
& \frac{d C_{\mathrm{co}_{2}}}{d t}=k_{\mathrm{co}_{2}} C_{\mathrm{o}}^{2}-\left(k_{\mathrm{co}_{2}}+k_{\mathrm{co}}\right) C_{\mathrm{co}_{2}}+k_{\mathrm{co}}^{\prime \prime \prime} C_{\mathrm{co}}^{2}+\lambda_{\mathrm{co}_{2}} \\
& \frac{d C_{\mathrm{co}}}{d t}=2 k_{\mathrm{co}} C_{\mathrm{co}_{2}}-2 k_{\mathrm{co}}^{m} C_{\mathrm{co}}^{2}+\lambda_{\mathrm{co}}-k_{\mathrm{co}}^{\prime} C_{\mathrm{co}}
\end{aligned}
$$

mit den anfangsbedingungen

$$
C_{\mathrm{o}}(o)=0 \quad C_{\mathrm{co}_{2}}(o)=0 \quad C_{\mathrm{co}}(o)=0
$$

G1(9-1) ist eine einfache Riccatische Differentialgleichung, aber anderseits ist es ziemlich kompliziert, die genauen Lösungen von $\mathrm{Gl}(9-2)$ und $\mathrm{G}_{1}(9-3)$, in denen $C_{\mathrm{o}}$ durch die Lösung von G1(9-1) bereits eliminiert wird, zu erhalten. Nimmt man aber eine Näherung

$$
k_{\mathrm{co}_{2}}^{\prime}=k_{\mathrm{co}}^{\prime} \equiv k,
$$


an, so wird folgende Lösung nach der in Anhang 3 gezeigten Berechnung durch den Ausdruck der Besselschen Funktion gegeben.

$$
\begin{aligned}
& C_{\mathrm{co}}=U_{\mathrm{co}}-Z_{\mathrm{co}} \\
& C_{\mathrm{co}_{2}}=A\left\{1-\exp \left(-k^{\prime} t\right)\right\}-\frac{U_{\mathrm{co}}-Z_{\mathrm{co}}}{2}
\end{aligned}
$$

wenn

$$
\begin{aligned}
& U_{\mathrm{co}}=\frac{B-\left(k^{\prime}+k_{\mathrm{co}}\right)}{4 k^{\prime \prime \prime}{ }_{\mathrm{co}}} \\
& Z_{\mathrm{co}}=\frac{-k^{\prime}}{4 k_{\mathrm{co}}} \cdot \frac{C_{1}\left\{2 \nu J_{\nu}(x)-x J_{\nu+1}(x)\right\}-C_{2} x J_{-\nu+1}(x)}{C_{1} J_{\nu}(x)+C_{2} J_{-\nu}(x)} \\
& C_{1}=\frac{\left(k^{\prime}+k_{\mathrm{co}}-B\right) J_{-\nu}(2 \sqrt{\beta})+k^{\prime} \sqrt{\beta} J_{-\nu+1}(2 \sqrt{\beta})}{\Delta k} \\
& C_{2}=\frac{-\left(k^{\prime}+k_{\mathrm{co}}+B\right) J_{\nu}(2 \sqrt{\beta})-k^{\prime} \sqrt{\beta} J_{\nu+1}(2 \sqrt{\beta})}{\Delta k^{\prime}} \\
& \Delta=J_{\nu}(2 \sqrt{\beta})\left[\nu I_{-\nu}(2 \sqrt{\beta})+2 \sqrt{\beta} J_{-\nu+1}(2 \sqrt{\beta})\right]+J_{-\nu}(2 \sqrt{\beta})\left[\nu J_{\nu}(2 \sqrt{\beta})-2 \sqrt{\beta} J_{\nu+1}(2 \sqrt{\beta})\right] \\
& x=2 \sqrt{\beta} \exp \left(-\frac{k^{\prime} t}{2}\right), \quad B=\frac{\sqrt{k^{2}\left(k^{\prime}+k_{\mathrm{co}}\right)^{2}+8 k^{\prime} k_{\mathrm{co}}^{\prime \prime}\left\{k_{\mathrm{co}}\left(i \gamma+2 \lambda_{\mathrm{co}_{2}}\right)+\lambda_{\mathrm{co}}\left(k^{\prime}+k_{\mathrm{co}}\right)\right\}}}{k^{\prime}} \\
& A=\frac{k_{\mathrm{co}}\left(i \gamma+2 \lambda_{\mathrm{co}_{2}}+\lambda_{\mathrm{co}}\right)}{k^{\prime}} \quad \beta=\frac{2 A \mathrm{k}_{\mathrm{co}}^{\prime \prime \prime}}{\mathrm{k}^{\prime 2}} \quad \nu=-\frac{\mathrm{B}}{\mathrm{k}^{\prime}}
\end{aligned}
$$

Hieraus folgt, daß die Reaktionspolarisation bei Prozeß (II-v) durch den Ausdruck

$$
\eta_{r}=\frac{R T}{8} \frac{T}{F_{r}}\left[\ln \frac{C_{\mathrm{co}_{2}}}{\left.9 C^{* 2}+\ln \frac{C_{\mathrm{co}}}{3 C^{*}}\right]}\right.
$$

dargestellt wird. Der Ausdruck der oben erhaltenen Lösung, in der die Werte jeder angegebenen Konstanten bzw. Koeffizienten in der Tat jedoch noch nicht ermittelt sind, ist aber zu kompliziert, als dass er zur Reurteilung der Polarisation-Zeit-Kurven praktisch anzuwenden wäre. Aus diesem Grund handelt es sich dabei besser um eine spezielle Bedingung, um die Eigenschaft dieses Prozesses irgendwie möglichst deutlich darzustellen.

Nun nehmen wir an, daß die Rückreaktion $\left(\mathrm{CO}_{2} \stackrel{k \text { " co }}{\longrightarrow} \mathrm{CO}\right)$ im Vergleich zur Hinreaktion $\left(\mathrm{CO}_{2} \stackrel{k \mathrm{co}}{\longrightarrow} \mathrm{CO}\right)$ sehr langsam und $\mathrm{zu}$ vernachlässigen ist. Dabei gelten die linearen Differentialgleichungen

$$
\frac{d C_{\mathrm{co}_{2}}}{d t}=\frac{i \gamma}{2}-\left(k_{\mathrm{Co}_{2}}^{,}+k_{\mathrm{co}}\right) C_{\mathrm{co}_{2}}+\lambda_{\mathrm{co}_{2}}
$$




$$
\frac{d C_{\mathrm{co}}}{d t}=2 k_{\mathrm{co}} C_{\mathrm{co}_{2}}-k_{\mathrm{co}} C_{\mathrm{co}}+\lambda_{\mathrm{co}}
$$

Hieraus ergeben sich

$$
C_{\mathrm{co}_{2}}=\frac{\left(i \gamma+2 \lambda_{\mathrm{co}_{2}}\right)}{2\left(k_{\mathrm{co}_{2}}^{\prime}+k_{\mathrm{co}}\right)}\left[1-\exp \left\{-\left(k_{\mathrm{co}_{2}}^{\prime}+k_{\mathrm{co}}\right) t\right\}\right]
$$

$$
\begin{aligned}
C_{\mathrm{co}}= & \frac{k_{\mathrm{co}}\left(i \gamma+2 \lambda_{\mathrm{co}_{2}}\right)+\lambda_{\mathrm{co}}\left(k_{\mathrm{co}_{2}}^{\prime}+k_{\mathrm{co}}\right)}{k_{\mathrm{co}}^{\prime}\left(k_{\mathrm{co}_{2}}^{\prime}+k_{\mathrm{co}}\right)}\left\{1-\exp \left(-k_{\mathrm{co}}^{\prime} t\right)\right\}+\frac{k_{\mathrm{co}}\left(i \gamma+2 \lambda_{\mathrm{co}_{2}}\right)}{\left(k_{\mathrm{co}}^{\prime}-k_{\mathrm{co}_{2}}^{\prime}-k_{\mathrm{co}_{0}}\right)\left(k_{\mathrm{co}_{2}}^{\prime}+k_{\mathrm{co}}\right)} \\
& \cdot\left[\exp \left(-k_{\mathrm{co}}^{\prime} t\right)-\exp \left\{-\left(k_{\mathrm{co}_{2}}^{\prime}+k_{\mathrm{co}}\right) t\right\}\right]
\end{aligned}
$$

und folglieh

$$
\begin{aligned}
\eta_{r}= & \frac{R T}{8 F_{r}} \ln \left[-\frac{\left(i \gamma+2 \lambda_{\mathrm{co}_{2}}\right)}{18 C^{* 2}\left(k_{\mathrm{co}_{2}}^{\prime}+k_{\mathrm{co}}\right)}\left\{1-\exp \left(-\left(k_{\mathrm{co}_{2}}^{\prime}+k_{\mathrm{co}}\right) t\right)\right\}\right] \\
& +\frac{R T}{4 F_{r}} \ln \left[\frac{\lambda_{\mathrm{co}\left(k_{\mathrm{co}_{2}}^{\prime}+k_{\mathrm{co}}\right)+k_{\mathrm{co}}\left(i \gamma+2 \lambda_{\mathrm{co}_{2}}\right)}^{3 C^{*} k_{\mathrm{co}}^{\prime}\left(k_{\mathrm{co}_{2}}^{\prime}+k_{\mathrm{co}}\right)}\left\{1-\exp \left(-k_{\mathrm{co}}^{\prime} t\right)\right\}}{}\right. \\
& \left.+\frac{k_{\mathrm{co}}\left(i \gamma+2 \lambda_{\mathrm{co}_{2}}\right)}{3 C *\left(k_{\mathrm{co}}^{\prime}-k_{\mathrm{co}_{2}}-k_{\mathrm{co}}\right)\left(k_{\mathrm{co}_{2}}^{\prime}+k_{\mathrm{co}}\right)}\left\{\exp \left(-k_{\mathrm{co}}^{\prime} t\right)-\exp \left(-\left(k_{\mathrm{co}_{2}}^{\prime}+k_{\mathrm{co}}\right) t\right)\right\}\right]
\end{aligned}
$$

Wenn man den Einfluß der Rückreaktion dabei berücksichtigen will, kann man von der Annahme ausgehen, dal3i es sich um eine Reaktion 1. Ordnung handelt; wenn man weiter noch die Annahme

$$
\mathrm{k}_{\text {co }}^{\prime}=\mathrm{k}_{\text {co }}^{\prime} \equiv \mathrm{k}^{\prime}
$$

macht, so gelten die Differentialgleichungen

$$
\begin{aligned}
& \frac{d C_{\mathrm{co}_{2}}}{d t}=\frac{i y}{2}-\left(k^{\prime}+k_{\mathrm{co}}\right) C_{\mathrm{co}_{2}}+k_{\mathrm{co}}^{m} C_{\mathrm{co}}+\lambda_{\mathrm{co}_{2}} \\
& \frac{d C_{\mathrm{co}}}{d t}=2 k_{\mathrm{co}} C_{\mathrm{co}_{2}}-\left(2 k_{\mathrm{co}}^{m}+k^{\prime}\right) C_{\mathrm{co}}+\lambda_{\mathrm{co}}
\end{aligned}
$$

Die Berechnung läßt sich verhältnismåßig leicht ausführen, nämlich

$$
\begin{gathered}
C_{\mathrm{co}_{2}}=\frac{k^{\prime}\left(i \gamma+2 \lambda \mathrm{co}_{2}\right)+2 k_{\mathrm{co}}^{\prime \prime}\left(i \gamma+2 \lambda_{\mathrm{co}_{2}}+\lambda_{\mathrm{co}}\right)}{2 k^{\prime}\left(k^{\prime}+k_{\mathrm{co}}+2 k_{\mathrm{co}}^{\prime \prime}\right)}\left\{1-\exp \left(-\left(k^{\prime}+k_{\mathrm{co}}+2 k_{\mathrm{co}}^{\prime \prime}\right) t\right)\right\} \\
\frac{k_{\mathrm{co}}^{\prime \prime}\left(i \gamma+2 \lambda_{\mathrm{co}_{2}}+\lambda_{\mathrm{co}}\right)}{k^{\prime}\left(k_{\mathrm{co}}+2 k_{\mathrm{co}}^{\prime \prime}\right)}\left\{\exp \left(-k^{\prime} t\right)-\exp \left(-\left(k^{\prime}+k_{\mathrm{co}}+2 k_{\mathrm{co}}\right) t\right)\right\}
\end{gathered}
$$




$$
\begin{aligned}
C_{\mathrm{co}}= & \frac{i \gamma+2 \lambda_{\mathrm{co}_{2}}+\lambda^{\mathrm{co}}\left\{1-\exp \left(-k^{\prime} t\right)\right\}}{k^{\prime}} \\
& -\frac{k^{\prime}\left(i \gamma+2 \lambda_{\mathrm{co}_{2}}\right)+2 k_{\mathrm{co}}^{m}\left(i \gamma+2 \lambda_{\mathrm{co}_{2}}+\lambda_{\mathrm{co}}\right)}{k^{\prime}\left(k^{\prime}+k_{\mathrm{co}}+2 k_{\mathrm{co}}^{\prime \prime}\right)}\left\{1-\exp \left(-\left(k^{\prime}+k_{\mathrm{co}}+2 k_{\mathrm{co}}^{\prime \prime}\right) t\right)\right\}+ \\
& +\frac{2 k_{\mathrm{co}}^{m}\left(i \gamma+2 \lambda_{\mathrm{co}_{2}}+\lambda_{\mathrm{co}}\right)}{k^{\prime}\left(k_{\mathrm{co}}+2 k_{\mathrm{co}}^{\prime m}\right)}\left\{\exp \left(-k^{\prime} t\right)-\exp \left(-\left(k^{\prime}+k_{\mathrm{co}}+2 k_{\mathrm{co}}^{m}\right) t\right)\right\}
\end{aligned}
$$

Damit erhält man ein $M a ß$ für den Einfluß der Rückreaktion auf die Polarisation. G1(9-15) und $\mathrm{G} 1(9-16)$ sind für $k_{\mathrm{co}}^{m}=0$ gleich zu G1(9-12), und G1(9-13) entsprechend für $k_{\mathrm{co}}^{\prime}=$ $k_{\mathrm{co}_{2}}^{\prime} \equiv \mathrm{k}^{\prime}$. Bei sehr kleinem $k_{\text {co }}$ (nämlich $k_{\text {co }}^{m} \rightarrow \infty$ ) ergibt sich $\mathrm{G} 1(6-7)$ aus G1(9-15), dabei ist $\mathrm{C}_{\text {co }}$ gleich Null.

(10) Der Fall, daß unmittelbar gebildetes $\mathrm{CO}$ an der Elektrode nach der bekannten Gleichgewichtsreaktion in $\mathrm{CO}_{2}$ übergeht, nämlich

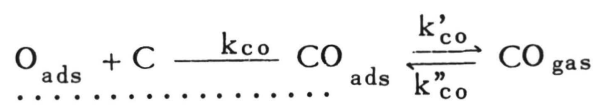

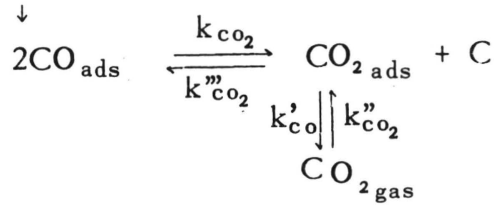

Für diese Aufgabe benutzt man dann die Differentialgleichungen

$$
\begin{aligned}
& \frac{d C_{\mathrm{o}}}{d t}=i \gamma-k_{\mathrm{co}} C_{\mathrm{o}} \quad(10-1) \quad \frac{d C_{\mathrm{co}}}{d t}=k_{\mathrm{co}} C_{\mathrm{o}}-2 k_{\mathrm{co}_{2}} C_{\mathrm{co}}^{2}-k_{\mathrm{co}}^{\prime} C_{\mathrm{co}}+2 k_{\mathrm{co}_{2}}^{\prime \prime} C_{\mathrm{co}_{2}}+\lambda_{\mathrm{co}} \\
& \frac{d C_{\mathrm{co}_{2}}}{d t}=k_{\mathrm{co}_{2}} C_{\mathrm{co}^{2}}^{2}-\left(k_{\mathrm{co}_{2}}^{\prime \prime}+k_{\mathrm{co}_{2}}^{\prime}\right) C_{\mathrm{co}_{2}}+\lambda_{\mathrm{co}_{2}}
\end{aligned}
$$

mit den Angangsbedingungen

$$
C_{\mathrm{o}}(o)=0 \quad C_{\mathrm{co}}(o)=0 \quad C_{\mathrm{co}_{2}}(o)=0
$$

Eliminiert man $C_{0}$ in obigen Differetialgleichungen, so hat man sie in der gleicliem Form wie G1(A2-1) und (A2-2). Nun nehmen wir aus dem oben beschriebenen Grund an, da 3 wir $\mathrm{K}_{\mathrm{co}_{2}}$ vernachlässigen können. Dann gelten die Riccatischen Gleichungen, die ohne Schwierigkeit unmittelbar zu lösen sind.

$$
\frac{d C_{\mathrm{co}}}{d t}=i \gamma+\lambda_{\mathrm{co}}-k_{\mathrm{co}}^{\prime} C_{\mathrm{co}}-2 k_{\mathrm{co}_{2}} C_{\mathrm{co}}^{2} \quad(10-5) \quad \frac{d C_{\mathrm{co}_{2}}}{d t}=\lambda_{\mathrm{co}_{2}}-k_{\mathrm{co}_{2}}^{\prime} C_{\mathrm{co}_{2}}+k_{\mathrm{co}_{2}} C_{\mathrm{co}}^{2}
$$

Die Löusungen ergeben sich daraus in der Gestalt:

$$
C_{\mathrm{co}}=U_{\mathrm{co}} \frac{\left\{2 k_{\mathrm{co}_{2}} U_{\mathrm{co}}\left(k^{\prime}+4 k_{\mathrm{co}_{2}} U_{\mathrm{co}}\right)-1\right\}\left\{1-\exp \left(\frac{-t}{k^{\prime}+4 k \mathrm{co}_{2} U_{\mathrm{co}}}\right)\right\}}{2 k_{\mathrm{co}_{2}} U_{\mathrm{co}}\left(k^{\prime}-4 k_{\mathrm{co}_{2}} U_{\mathrm{co}}\right)\left\{1-\exp \left(\frac{-t}{k^{\prime}+4 k_{\mathrm{co}_{2}} U_{\mathrm{co}}}\right)\right\}-1}
$$


$C_{\mathrm{co}_{2}}=\frac{\left(i \gamma+\lambda_{\mathrm{co}}+2 \lambda_{\mathrm{co}_{2}}\right)\left\{1-\exp \left(-k^{\prime} t\right)\right\}}{2 k}-\frac{C_{\mathrm{co}}}{2}$

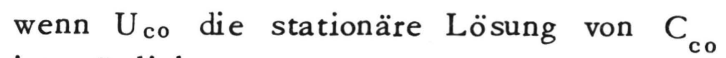
ist, nämlich

$$
U_{\mathrm{co}}=\frac{-k^{\prime}+\sqrt{k^{\prime 2}+8 k_{\mathrm{co}_{2}}\left(i \gamma+\lambda_{\mathrm{co}}\right)}}{2 k_{\mathrm{co}_{2}}}
$$

wobei wir annehmen

$$
k_{\mathrm{co}}^{\prime}=k_{\mathrm{CO}_{2}}^{\prime} \equiv k_{\mathrm{o}}^{\prime}
$$

$\mathrm{Da}$ diese Lösungen zur praktischen Analyse unserer Aufgabe immer noch etwas zu kompliziert sind, nehmen wir zur Reduzierung des Ausdrucks der Lösungen an, daß die Reaktionen dabei nach der ersten Ordnung gehen. Dadurch werden die Gl(10-5) und G1(10-6) in folgender Gestalt dargestellt

$$
\begin{aligned}
& \frac{d C_{\mathrm{co}}}{d t}=i \gamma+\lambda_{\mathrm{co}}-\left(k_{\mathrm{co}}^{\prime}+2 k_{\mathrm{co}_{2}}\right) C_{\mathrm{co}} \\
& \frac{d C_{\mathrm{co}_{2}}}{d t}=\lambda_{\mathrm{co}_{2}}-k_{\mathrm{CO}_{2}}^{\prime} C_{\mathrm{CO}_{2}}+k_{\mathrm{co}_{2}} C_{\mathrm{co}}
\end{aligned}
$$

Hieraus ergeben sich die Lösungen

$$
C_{\mathrm{co}}=\frac{i \gamma+\lambda_{\mathrm{co}}}{k_{\mathrm{co}}^{\prime}+2 k_{\mathrm{co}_{2}}}\left[1-\exp \left\{-\left(k^{\prime}+2 k_{\mathrm{co}_{2}}\right) t\right\}\right]
$$

$C_{\mathrm{co}_{2}}=\frac{\lambda_{\mathrm{Co}_{2}}\left(k_{\mathrm{co}}^{\prime}+2 k_{\mathrm{co}_{2}}\right)+k_{\mathrm{co}_{2}}\left(i \gamma+\lambda_{\mathrm{co}_{0}}\right)}{k_{\mathrm{Co}_{2}}^{\prime}\left(k_{\mathrm{co}_{\mathrm{o}}}^{\prime}+2 k_{\mathrm{co}_{2}}\right)}$

$$
\times\left\{1-\exp \left(-k_{\mathrm{Co}_{2}}^{\prime} t\right)\right\}
$$

$$
\begin{aligned}
& +\frac{k_{\mathrm{co}_{2}}\left(i \gamma+\lambda_{\mathrm{co}}\right)}{\left(k_{\mathrm{co}_{2}}^{\prime}-k_{\mathrm{co}_{0}}^{\prime}-2 k_{\mathrm{co}_{2}}\right)\left(k_{\mathrm{co}}^{\prime}+2 k_{\mathrm{co}_{2}}\right)}\left\{\exp \left(-k_{\mathrm{co}_{2}}^{\prime} t\right)\right. \\
& \left.-\exp \left(-\left(k_{\mathrm{co}}^{\prime}+2 k_{\mathrm{co}_{2}}\right) t\right)\right\}
\end{aligned}
$$

$$
\begin{aligned}
& \eta_{r}=\frac{R T}{6 F_{r}}\left[\operatorname { l n } \left[\frac{i \gamma+\lambda_{\mathrm{co}}}{3 C^{*}\left(k_{\mathrm{co}}^{\prime}+2 k_{\mathrm{co}_{2}}\right)}\{1-\exp (-(k,\right.\right. \\
& \left.\left.\left.\left.+2 k_{\mathrm{co}_{2}}\right) t\right)\right\}\right] \\
& +\ln \left[\frac{\lambda_{\mathrm{Co}_{2}}\left(k_{\mathrm{Co}_{2}}^{\prime}+2 k_{\mathrm{co}_{2}}\right)+k_{\mathrm{co}_{2}}\left(i \gamma+\lambda_{\mathrm{co}_{0}}\right)}{9 C^{* 2} k_{\mathrm{Co}_{2}}^{\prime}\left(k_{\mathrm{co}}^{\prime}+2 k_{\mathrm{co}_{2}}\right)}\right.
\end{aligned}
$$$$
\times\left\{1-\exp \left(-k_{\mathrm{CO}_{2}} t\right)\right\}
$$

$$
+\frac{k_{\mathrm{co}_{2}}\left(i \gamma+\lambda_{\mathrm{co}}\right)}{9 C^{* 2}\left(k_{\mathrm{co}_{2}}^{\prime}-k_{\mathrm{co}_{0}}^{\prime}-2 k_{\mathrm{co}_{2}}\right)\left(k_{\mathrm{co}}^{\prime}+2 k_{\mathrm{co}_{2}}\right)}
$$

$\left.\left.\times\left\{\exp \left(-k_{\mathrm{Co}_{2}}^{\prime} t\right)-\exp \left(-\left(k_{\mathrm{co}}^{\prime}+2 k_{\mathrm{co}_{2}}\right) t\right)\right\}\right]\right]$

\section{Nichtfaradayscher Strom}

Bei den bisher erhaltenen theoretischen Gleichungen für die Polarisation-ZeitKurven bei konstanter Stromdichte geht man von der Annahme aus, daß die Konzentration der oxydierten Stoffe wegen der Benutzung einer Fremdelektrode gleich nach dem Einschalten in der Nähe von Null liegt. Daher kommen die theoretischen Kurven aus dem Negativ-Unendlichen und steigen dann wie in Abb. 4(a) gezeigt an. Aber der wirkliche zeitliche Polarisationsverlauf beginnt ohne Ausnahme immer unmittelbar im Positiven, wie aus Abb. 4(b) hervorgeht. Das Verhalten der Fremd elektrode gegenüber dem Finschalten läßt sich am einfachsten an Hand des in Abb. 5 gegebenen Ëquivalentschal tbildes übersehen, $\mathrm{R}$ ist der Elektrolytwiderstand, der in Serie mit der Doppelschichtkapazität $\mathrm{C}_{\mathrm{d}}$ und einem ihr parallel geschalteten Polarisationsteil liegt. Der letztere wird allerdings in einen EMK-Teil $(\epsilon+\eta(t))$ bezuglich der Polarisation, und einen ohmschen Widerstand $\mathrm{Re}_{\mathrm{e}}$ (an der Grenz $\mathrm{z}$ wisschen der Elektrodenoberfläche und dem Elektrolyt) abgeteilt, wenn $\epsilon$ das Gleichgewichtspotential zwischen der Fremdelektrode und dem Elektrolyt ist. Bezeichnet 
man die Ströme,

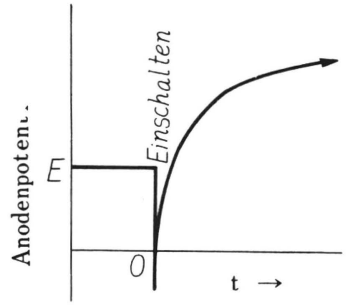

(a)

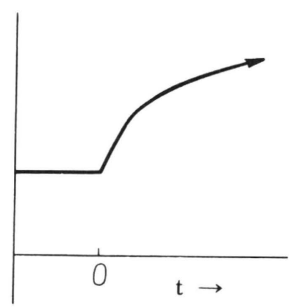

(b)
Abb. 4

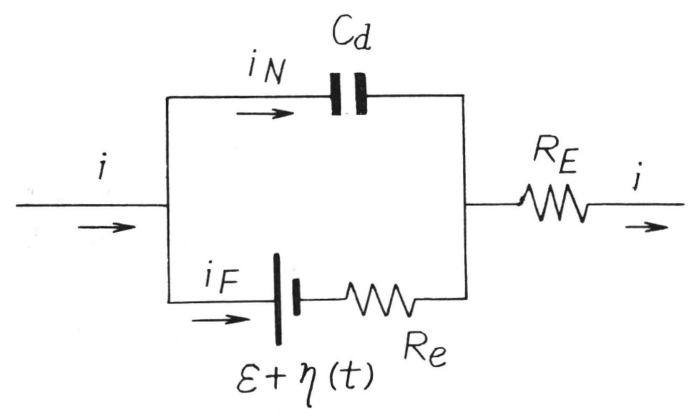

Abb. 5

die durch jeden Zweig fließen, als nichtfaradayschen Strom $i_{\mathrm{N}}$ und Faradayschen Strom $i_{\mathrm{F}}$, dann gilt für Kreisschaltung ABA die Gleichung

$$
\frac{1}{C_{d}} \int i_{\mathrm{N}} d t-R_{e} i_{\mathrm{F}}=\epsilon+\eta(t)
$$

$\mathrm{R}_{\mathrm{e}}$ ist zwar vielleicht praktisch nicht ganz konstant, wiel die Eigenschaft der Elektrodenoberfläche sehr athängig von den daran abgeschiedenen Stoffen ist. Nimmt man aber $R_{\mathrm{e}}=$ konstant an, so erhalten wir aus $\mathrm{Gl}(11-1)$
$\frac{1}{C_{d}} \int i_{\mathrm{F}} d t+R_{e} i_{\mathrm{F}}=\frac{1}{C_{d}}-\{\epsilon-\eta(t)\}$

weil

$i_{\mathrm{F}}+i_{\mathrm{N}}=i($ konstan $\mathrm{t})$

Hieraus ergibt sich $i_{F}$

$$
\begin{aligned}
& i_{F}=i-\frac{1}{R_{e}} \exp \left(\frac{-t}{R_{e} C_{d}}\right) \int \frac{d \eta}{d t} \exp \left(\frac{t}{R_{e} C_{d}}\right) d t \\
& -i_{F_{0}} \exp \left(\frac{-t}{R_{e} C_{d}}\right)
\end{aligned}
$$

wenn $i_{\mathrm{F}}$ die Integralkonstante ist. Bei $t=0$ (vor dem Einschalten) sind selbstverständig $i, i_{\mathrm{F}}$ und $\eta(t)$ gleich Null, daher ist auch $i_{\mathrm{F}_{0}}=$ o. Setzt man nun z.B.

$$
\eta=\frac{R T}{n F_{r}} \ln [k\{1-\exp (-k t)\}]
$$

aus $\mathrm{Gl}(5-7)$ voraus, und nimmt man noch $k \gg \frac{1}{R_{\mathrm{e}} \mathrm{C}_{\mathrm{d}}}$ an, so ergibt sich

$$
i_{F}=i-\frac{\eta}{R_{e}} \exp \left(\frac{-t}{R_{e} C_{d}}\right)
$$

d.h. der Faradaysche Strom ist trotz des konstanten Gesamtelektrodenstromes am kleinsten bei $t=0$ und wird mit der Zeit größer. Das beobachtete Elektrodenpotential ist

$$
E=\left(R_{E}+R_{e}\right) i+\epsilon+\eta\left\{1-\exp \left(-\frac{-t}{R_{e} C_{d}}\right)\right\}
$$

Bezeichnet man die beobachtete Polarisation mit $\eta^{\prime}$, so verliält sie sich zur theoretischen Polarisation $\eta$ wie Gl(11-6) zeigt.

$$
\eta^{\prime}=\eta\left\{1-\exp \left(-\frac{-t}{R_{e} C_{d}^{-}}\right)\right\}
$$

Abb. 6 zeigt ihre graphische Darstellung für kleine Zeiten. Hier ist die Doppelschichtkapazität, also der Nichtfaradaysche Storm, potentialbestimmend. Beurteilt man beitgrösser Zeit der Grösserordnung von mi lisekundären die Polarisationskurven, 


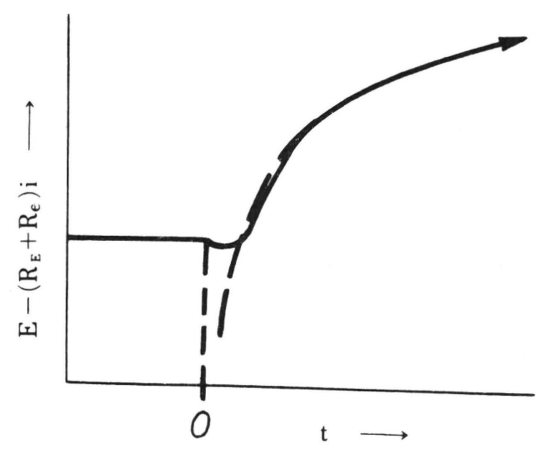

Abb. 6

so kann man sicher den nichtfaradayschen Strom vernachlässigen, da der Glied exp $\left(\frac{t}{R_{\mathrm{e}} C_{\mathrm{d}}}\right)$ bei $t>B_{\mathrm{e}} C_{\mathrm{d}}$ verschwindet.

\section{Versuchsordnung}

In Abb. 7 sind das Schaltschema der Meßanordung: und der Aufbau der Apparatur skizziert.

Die Impedanz der Elektrolysezelle ändert sich während des Versuchs. Um nun an der Zelle immer eine möglichst konstante Stromstärke zu haben, mußte eine Stromquelle großer Leistung benutzt werden, Wir hatten einen $150 \mathrm{~V}$ Gleichrichter zur Verfügung der $20 \mathrm{~A}$ lieferte. Der Gleichstrom wurde durch eine Siebkette geglättet, hinter die ein Vorschaltwiderstand für hohe : Leistung gelegt wurde. Dadurch wurde zugleich verhindert, da $\beta$ die beim Einschalten der Apparatur auftretenden Schwingungen die Messungstören konnten. Die gesamte Meßanordnung wurde zuerst in Leerversuchen geeicht (wobei die Elektrolysezelle durch einen Widerstand (ca.3 3 ) ersetzt war). Der Elektrolysestrom war höchstens ca.0,5A für die gemessene max. Anodenstromdichte; deswegen kann man für die Messung der Potential-Zeit-Kurven mit genügender $\mathrm{Ge}$ nauigkeit konstanten Strome annehmen. Der Spannungsabfall an der Zelle betrug höch-

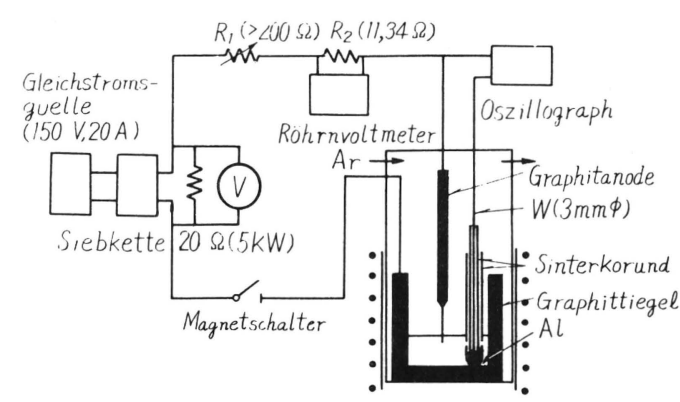

Abb. 7

stens 2 Volt. Um keine Konvektion auftreten zu lassen, wurde kein Tyratronschalter, sondern von Handein- und ausgeschaltet und die Apparatur nur kurze Zeiten (max. $1 \mathrm{sec}$ ) eingeschaltet gelassen.

Für die Elektrolyse diente eine vollkommenen geschlossene Apparatur, wie sie sich bei unseren bisherigen Versuchen als die beste erwiesen hatte. Durch Füllen der Apparatur mit Argon konnte für eine völlig indiffernte Atmosphäre gesorgt werden. Als Anode diente ein Graphitstab von $0,6 \mathrm{~cm} \phi \times$ $30 \mathrm{~cm}$ der Siemens-Plania-Werke, der am Eintauschende $5 \mathrm{~cm}$ lang auf $2 \mathrm{~mm} \phi$ abgedreht war, die Eintauschliefe war ca $8 \mathrm{~mm}$. Als Bezugselektrode diente ein in geschmolzenes Aluminium tauschender Wolframstab, der durch ein Sinterkorundrohr geschützt war. Die ganze Bezugselektrode war in einem unten geschlossenen Sinterkorundrohr mit 2 seitlichen Bohrungen untergebracht. Die Elektrolyse wurde bei $870^{\circ} \mathrm{C}$ in einem Elektrolyt von $\mathrm{Li}_{3} \mathrm{AlF}_{6}(30 \mathrm{Mo} 1 \%)-\mathrm{Na}_{3} \mathrm{AlF}_{6}(65 \mathrm{Mo} 1 \%)$ $\mathrm{Al}_{2} \mathrm{O}_{3}(5 \mathrm{Mol} \%)$ durchgeführt.

\section{Versuche}

Abb. 8 zeigt oszillographische Aufnahmen der Potentialkurven der Kohleanode bei verschiedenen Stromdichten.

Bei größeren Stromdichten (über ca. 300 $\mathrm{mA} / \mathrm{cm}^{2}$ ) und etwas längerer Zeit (über ca $200 \mathrm{msec}$ ) wurden unregelmäßige 


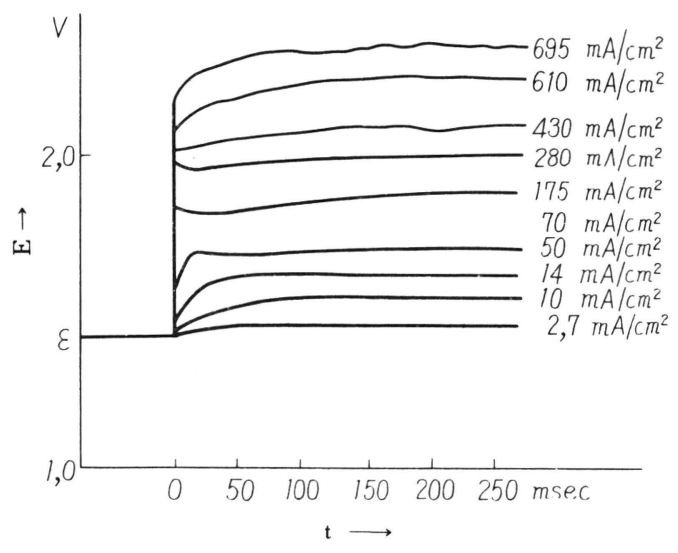

Abb. 8

gungen beobachtet, die wahrscheinlich von den entwickelten Gasblasen herstammen. Hier dürfte sich auch der Einfluß der Konvektion störend bemerkbar machen. Eine theoretische Analyse dieser Konvektion ist sicher sehr schwer, weil man in diesem nichtstationären System keine konstante hydrodinamische Geschwindigkeit annehmen kann. Freilich sollte man, wie aus Abb. 3 zu ersehen ist, für die Analyse der Diffusion trotz dieser Schwierigkeit möglichst die Kurven höherer Stromdichte benutzen, freilich unter möglichstern Vermeiden der Konvektion weil hier die Diffusionspolarisation nicht nur größer ist, sondern auch später den stationären Zustand erreicht.

Bei sehr nierdrigen Stromdichten scheint die Diffusionspolarisation vernachlässigeswert klein zu sein. Die Gesammtpolarisation hat nach längstens $50 \mathrm{msec}$ ihren stationären Wert erreicht, diese Zeit sinkt mit wach sender Stromdichte.

Wir haben die Kurven höherer Stromdichte zur Analyse der Diffusionspolarisation benutzt und die Kurven niedriger Stromdichte, nachdem die berechnet Diffusionspolarisation abgezogen worden war, zur Analyse der Reaktionspolarisation herangezogen.

\section{Diffusionspolarisation}

Es dürfte am besten sein, die Kurven der in der theoretischen Betrachtung erhaltenen verschiedenen Diffusionsgleichung en numerisch auszuwerten und mit den experimentellen Kurven zu vergleichen, um den diffusionspotentialbestimmenden Proze $\beta \mathrm{zu}$ erkennen, wenn die Konstanten $\mathrm{D}$ und $\delta$ mittels andrer Meßmethoden zu bestimmen wären. Aber leider ist es gegenwärtig noch unmöglich, weil die Bestimmung von $D$ und $\delta$ durch die schlechten Eigenschaften dieses Systems, z.B. die Undeutlichkeit des Grenzstroms, die starke Angriffsfähigkeit der Schmelze, die ziemlich hohe Temperatur u.s.w., verhindert ist. Wir haben daher umgekehrt aus den experimentellen Ergebnissen $\mathrm{D}$ und $\delta$ in den theoretischen Gleichungen unmittelbar bestimmt, und dann durch die Ermittelung der Werte dieser Konstanten den Prozeß ausgewählt in den die Stoffe in diesem Schmelzfluß an den Anodenvorgängen teilnehmen.

Für die Diskussion über die Diffusionspolarisation werden die in Abb. 9 gezeigten Potential-Zeit-Kurven nach den schon beschriebenen Bedingungen ausgewählt.

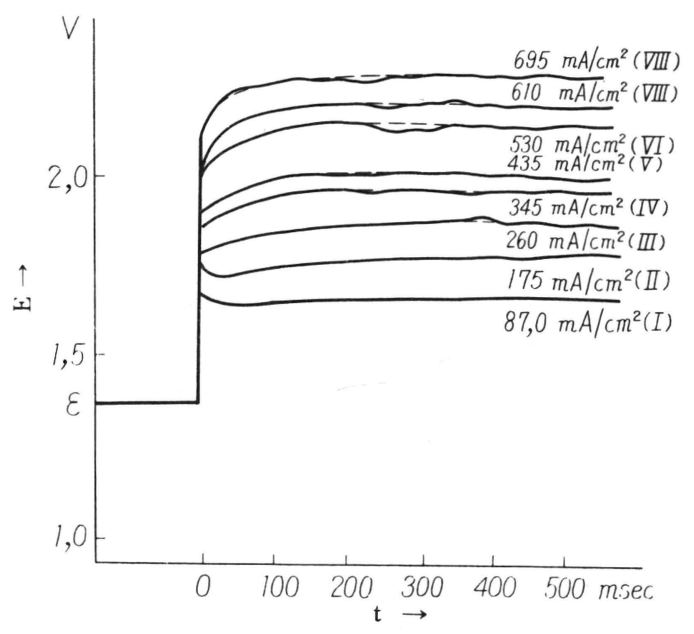

Abb. 9 
Allgemein verstehen wir, in der ersten Näherung wie schon in der Einleitung erwähnt, daß die aus den gemessenen Kurven unmittelbar beobachtete Polarisation

$$
\eta_{b}=\eta_{d}+\eta_{r}+\eta_{w}+R^{\prime} i
$$

genügt (Dabei haben wir genommen, da $\beta \eta_{\mathrm{D}}$ in Vergleichen $z u$ andren Gliedern sehr klein ist), wenn

$$
R^{\prime}=R_{E}+R_{e}
$$

Nehmen wir nun an, daß während der Zeit, für die die Daten der Gegenstand unserer Analyse sind, die Widerstandspolarisation $\eta_{\mathrm{w}}$ vermutlich konstant ist und die Reaktionspolarisation vielleicht schon den stationären Zustand erreicht hat, so kann man erkennen, daß $\eta_{\mathrm{d}}$ aus dem zu $t$ abhängigen Teil $\eta_{\mathrm{b}}$ und dem zu $t$ unabhängigen Teil $\left(\eta_{\mathrm{rs}}+\eta_{\mathrm{w}}+R^{\prime} i\right)$

zusammengesetzt, nämlich :

$$
\eta_{d}=\eta_{b}-\left(\eta_{r s}+\eta_{w}+R^{\prime} i\right)
$$

wobei $\eta_{\text {rs }}$ die stationäre Polarisation von $\eta_{\mathrm{r}}$ ist.

Zuerst ist $\mathrm{Gl}(3-6)$ erhalten worden, die unter der Annalime, daß bei dem Prozeß (I-vi) oder (I-iii), M seltene Ionen im Schmelzfluß sind,; Im Vergleich mit andren Gleichungen ist dies ein besonders spezieller Ausdruck. Aber es ist fraglich, ob $\mathrm{M}$ bei Prozess (I-iii) noch die diffusionsbestimmende spezielle Form lat, nachidem $M^{\mathrm{n}} \mathrm{i}$ das Sauerstoff bei dem Elektrodenprozess verloren hat. Daher haben wir G1 (3-6) aus der folgenden Diskussion ausgeklammert. Die übrigen Differentialgleichungen sind allgemein mit folgendem Ausdruck

$$
\eta_{d}=-\frac{R T}{n F_{r}} \ln \left[1-\frac{i \delta}{6 D C^{*} F_{r}}\right.
$$

$$
\left.+\frac{4 i \delta}{3 D \pi^{2} C^{*} F_{r}} \sum_{0=u}^{\infty} \frac{\exp \left(\frac{-D \pi^{2}(1+2 n)^{2} t}{4 \delta^{2}}\right)}{(1+2 n)^{2}}\right]
$$

zu bezeichnen, mit $\mathrm{n}=2$ beim Prozeß (I-i), und (I-iii), $\mathrm{n}=4$ beim Prozeß (I-iv) und $\mathrm{n}=6$ beim = Prozeß (I-ii). Wird die stationäre Polarisation von $\eta_{\mathrm{d}}$ und $\eta_{\mathrm{b}}$ mit $\eta_{\mathrm{ds}}$ und $\eta_{\mathrm{bs}}$ bezeichnet, so ergibt sich aus G1 (12-2) und $\mathrm{Gl}(12-3)$ einfacl:

$$
\begin{aligned}
& \exp \left(-\frac{n F r}{R T} \eta_{b}\right)-\exp \left(-\frac{n F r}{R T} \eta_{b s}\right) \\
& =\frac{\frac{4 i \delta}{3 D \pi^{2} C^{*} F_{r}} \sum_{n=0}^{\infty} \frac{\exp \left(\frac{-D \pi^{2}(1+2 n)^{2} t}{4 F_{r}}\right)}{\exp \left(\frac{n \delta^{2}}{R T}\left(\eta_{r s}+\eta_{w}+R^{\prime} i\right)\right\}}}{(1+2 n)^{2}}
\end{aligned}
$$

weil

$$
\eta_{d s}=-\frac{R T}{n F_{r}} \ln \left[1-\frac{i \delta}{6 D C^{*} F_{r}}\right]
$$

ist. Ist $t$ genügend gro $\beta$ (z. B. $t>50 \mathrm{~m}$ sec bei $D=10^{-5} \mathrm{~cm}^{2} / \mathrm{sec}$ und $\left.\delta=10^{-3} \mathrm{~cm}\right)$,

$$
\begin{aligned}
& \log \left[\exp \left(-\frac{n F_{r}}{R T} \eta_{b}\right)-\exp \left(-\frac{n F_{r}}{R T} \eta_{b s}\right)\right] \\
& =\log \left(\frac{4 i \delta}{3 D \pi^{2} C^{*} F_{r}}\right)-0.434 \frac{n F r}{R T}\left(\eta_{r s}+\eta_{w}+R^{\prime} i\right) \\
& -0.434 \cdot \frac{D \pi^{2}}{4 \delta^{2}} t
\end{aligned}
$$

weil dabei ungefähr

$$
\sum_{n=1}^{\infty} \frac{\exp \left(\frac{-D \pi^{2}(1+2 N)^{2} t}{4 \delta^{2}}\right)}{(1+2 n)^{2}}=\exp \left(-\frac{D_{\pi^{2} t}}{4 \delta^{2}}\right)
$$

gibt.

Aus Grund von Gl(12-6) haben wir die gemessenen V'erte bzw. die daraus berechneten W'erte der linken Seite von Gl(12-6) 
gegen die Zeit aufgetragen (Abb.10). Nach $\mathrm{Gl}(12-6)$ soll man theoretisch bei verschiedenen

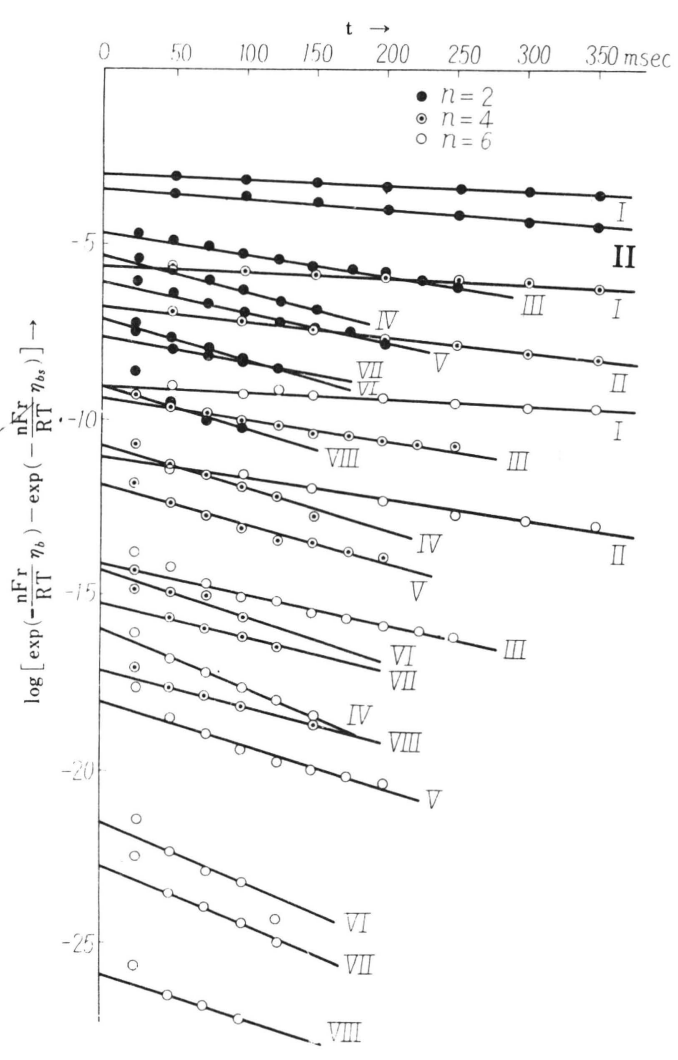

Abb. 10

Stromdichten die einander parallelen Geragen bekommen. Es wurden aber Abweichungen beobachtet, die daher rühıren, daß bei kleineren $t \mathrm{Gl}(12-7)$ nicht mehr ganz gilt und bei größern $i$ sich wahrscheinlich der Einfluß der Gasblasen bemerkbar macht. Beim letzteren Fall ist $\eta_{\mathrm{w}}$ offenbar nicht mehr konstant und $\delta$ wegen der Konvektion kleiner, und je gößer $\mathrm{i}$ ist, desto größer wird die Neigung der Geraden.

Trägt man die Neizung der Geraden gegen entsprechende Stromdicliten auf, und extrapoliert für $i \rightarrow 0$, vie Alt.11 zeigt, so ist der Wert $-0,434 \frac{D \pi^{2}}{4 \delta^{2}}$ für die reine Diffusion oline ronvektion zu bestimmen, nämlich 0,528 für $\frac{D}{\delta^{2}}$ bei $\mathrm{n}=2,0,620$ bei bei $n=4$ und 0,663 bei $n=6$.

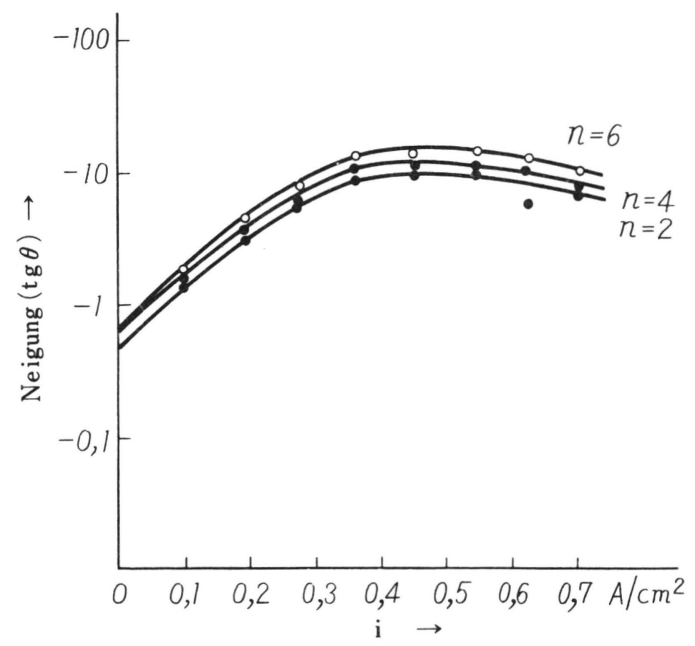

Abb. 11

Dann kann man aus dem Wert, der durch das Extrapolieren der Geraden zu $t \rightarrow 0$ in Abb.10 erhalten wurde.

$$
\log \left(\frac{4 i \delta}{3 D \pi^{2} C^{*} F r}\right)-0.434 \frac{n F r}{R T}\left(\eta_{\mathrm{rs}}+\eta_{\mathrm{w}}+R^{\prime} i\right)
$$

bestimmen. Nachdem wir die um $\log i$ verminderten Werte gegen $i$ aufgetragen hatten, haben wir sie zu $i \rightarrow 0$, wie oben, extrapoliert, wie in Abb. 12 gezeigt. Damit kann man unmittelbar den Wert von $\log \cdot \frac{4 \partial}{3 D \pi^{2} C^{*} F_{r}}$ bei reiner Diffusion finden, wenn $\eta_{\mathrm{w}}$ für die Anderung von $i$ das zu $\eta_{\mathrm{rs}}$ ähnliche Verhalten gibt, weil $\eta_{\mathrm{rs}}$ und $R^{\prime} i$ mit der Abnahme von $i$ sicher monoton abnehmen und

$$
\lim _{i \rightarrow 0}\left(\eta_{r s}+\eta_{w}+R^{\prime} i\right)=0
$$

ist. Für $\frac{\delta}{D}$ ergibtn sich hieraus $4,51 \times 10$ bei $n=2,3.87 \times 10^{-1}$ bei $\mathrm{n}=4$ und $1.55 \times 10^{-3}$ bei $n=6$. Schließlich haben wir nach der einfachen Berechnung 


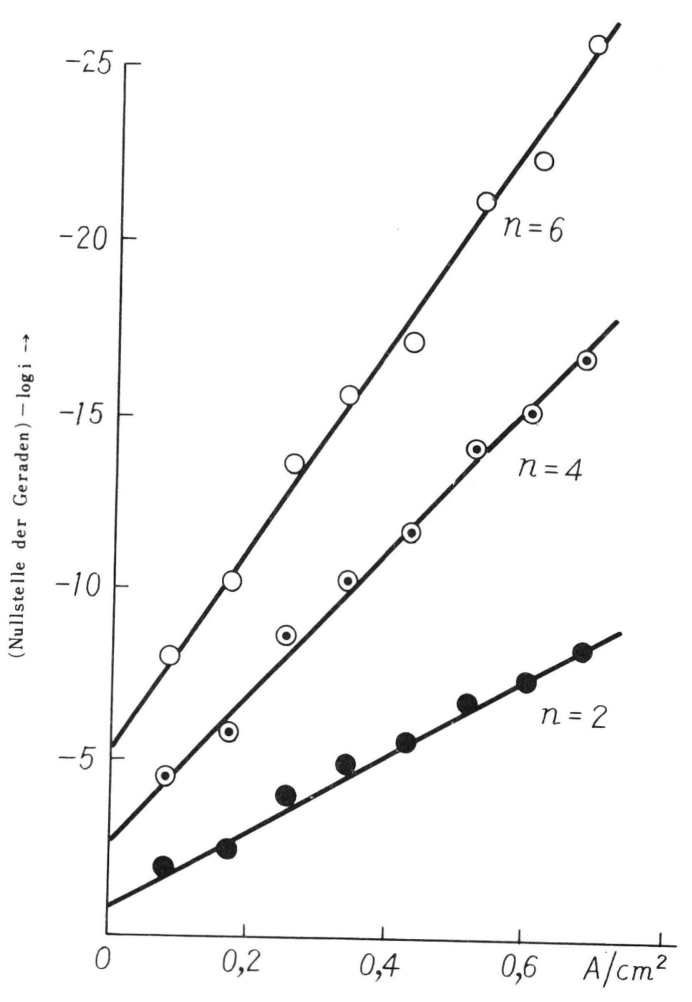

Abb. 12

auch mit vorliegenden Daten die Werte für $\delta$ und $D$ erhalten, nämlich $\delta=4.13 \cdot 10^{-2} \mathrm{~cm}$, $D=9.28 \cdot 10^{-4} \mathrm{~cm}^{2}$ sec bei $n=2, \delta=1.32 \mathrm{~cm}$, $D=2.05 \mathrm{~cm}^{2} / \mathrm{sec}$ bei $n=4$ und $\delta=9.68 \cdot 10^{2} \mathrm{~cm}$, $D=6.25 \cdot 10^{5} \mathrm{~cm}^{2} / \mathrm{sec}$ bei $n=6$. Die Werte bei $n=4$ und $n=6$ sind offensichtlich zu abnormal, selbst wenn verschiedene wahrscheinliche Einfluses berücksichtigt würden, deswegen kann man nun bestimmt entscheiden, daß $\mathrm{Al}_{2} \mathrm{O}_{3}$ selbst an der Anodenvergängen unmittelbar nicht teilnimmt.

Die Lestinmten. Ferte von $D$ und $\delta$ bei $n=2$ scheinen auch noch etwas zu gross zu sein, jedocl sind der Prozess (I-i) kzw. (I-iii) sehr wahrscheinlich, wenn man berücksichtigt, dass die schon beschriebenen Annäherungsmethoden für die Berechnung des Randbedingungsproblems und der Gesamm, tpolarisation angewandt wurden, dass die unregelmässige $\mathbb{W}$ iderstandspolarisation die Messergebnisse verfälscht hat und dass die Thermokonvektion im Elektrolyt vielleicht ziemlich gross ist.

Man sollte annahmen, dass die Abnahme der Widerstandspolarisation nicht monoton sondern stufenweise erfolgt, wie in Abb.13 gezeigt, weil sie in wesentlichen unmittelbar abhängig von der durch das Anodengas auf gebauten Deckschicht ist. Wenn dies so wäre, ist unsere Extrapolation bestimmt

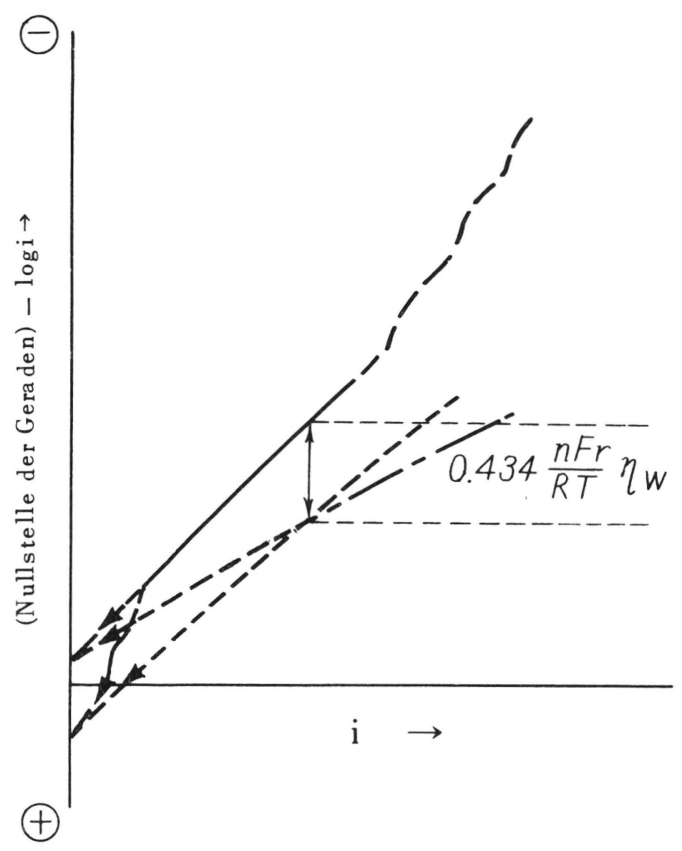

Abb. 13

nicht gut, weil die Kurve ungefähr parallel um $\eta_{\mathrm{w}}$ nach unten verschoben ist, wie die mit einer punktierten Linie gezeigte Kurve zeight. Wir hä tten daher sicher für $\frac{\delta}{\mathrm{D}}$ einer noch größeren Wert bekommen, wenn der Wert von $\eta_{\mathrm{w}}$ bekannt wäre. Damit ergäben sich noch kleineres $\delta$ und auch kleineres $D$, wie sie auch wahrscheinlicher sind.

Hiermit können wir zusammenfassen, daß bei der Elektrolyse von Kryolith $\mathrm{O}^{2-}$ Ionen bzw. Reste, die nur ein Sauerstoffatom enthalten, an den Anodenvorgänge sicher teilnehmen. 


\section{Reaktionspolarisation}

Aus dem vorher schon beschriebene Grund wurde die bei niedrigerer Stromdichte gemessenen Kurven für die Analyse der Reaktionspolarisation berücksichtigt, wie aus Abb. 14 hervorgeht. Aus der oben definierten Gleichung der Diffusionspolarisation $(n=2)$

$$
\begin{aligned}
& \eta_{d}=-0.114 \log \{1-0.126 i \\
& \left.+0.102 i \sum_{n=0}^{\infty} \frac{\exp \left\{-1.06(1+2 n)^{2} t\right\}}{(1+2 n)^{2}}\right\}
\end{aligned}
$$

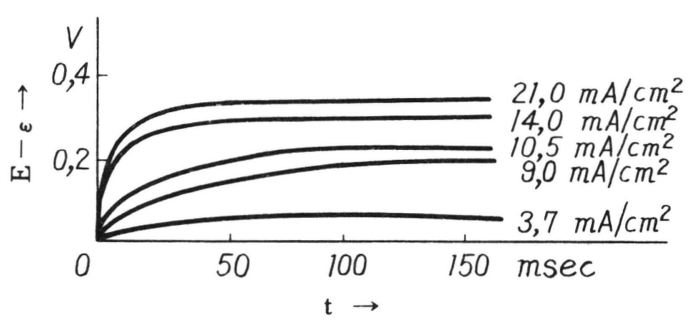

Abb. 14

ist die Diffusionspolarisation unmittelbar auszurechnen, wie in Abb. 15 wiederge-

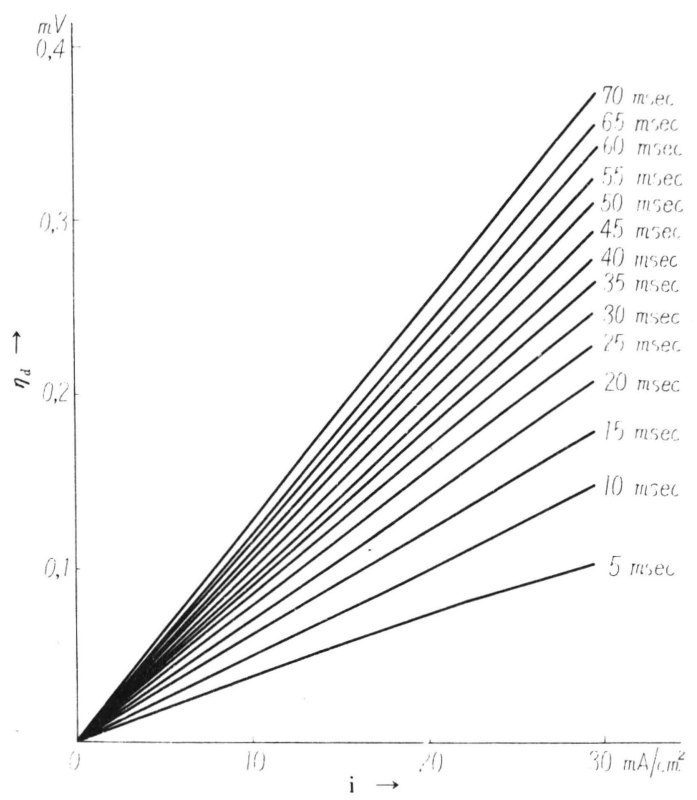

Abb. 15 geben. Man kann daran ersehen, daB die Diffusionspolarisation in Vergleich zu den zur Analyse dienenden Kurven zu vernachlässigen ist.

Der Ohmsche Widerstand zwischen der zu beobachtenden Anode und der Bezungselektrode wurde vorher mittels der Wechselstromsmethode gemessen. (Es wurde) zu $\omega \rightarrow \infty$ extrapoliert.) In Abb. 16 wurde die oscillographisch gemessene Ohmsche Spannung, die aus dem oben gemessenen Wert $2.79 \Omega / \mathrm{cm}^{2}$ (Anode)

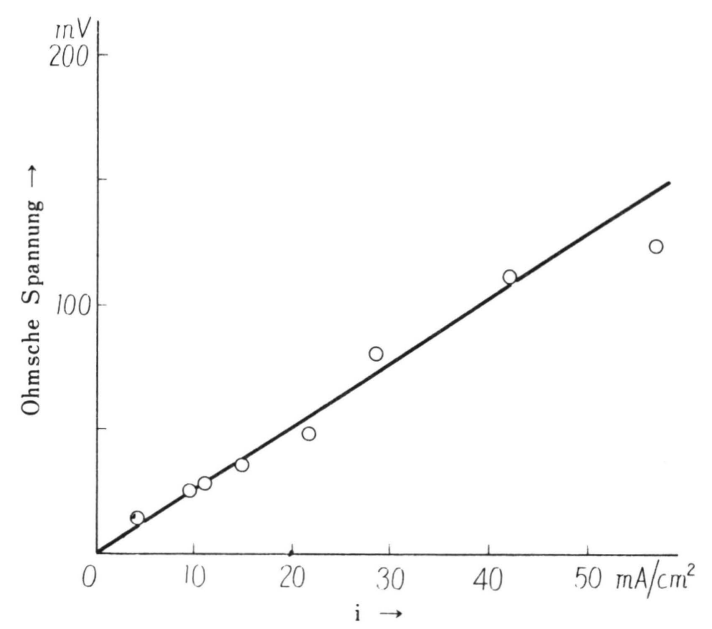

Abb. 16

ausgerechnet wurde, gegen die anodische Stromdichte aufgetragen. Wir haben den Ohmschen Widerstand, der zu $2.79 \Omega / \mathrm{cm}^{2}$ (Anode) berechnet wurde, von den Kurven der oszillographischen Messung subtrahiert, weil die berechneten Spannungen, trotz der ziemlich guten Übereinstimmung mit den gemessenen Werten genauer zu sein scheinen. Endlich wurde Abb. 17 dargestellt. $\quad\left(\eta_{\mathrm{r}}^{\prime}=\eta_{\mathrm{r}}+\eta_{\mathrm{w}}\right)$

Die in Abb. 17 wieder gegebenen Kurven sind jedoch nicht sehr praktisch, um zur Analyse zu dienen. Der Unterschied mit der stationären Polarisation wurde dabei gegen der Stromdichten aufgetragen, wie 


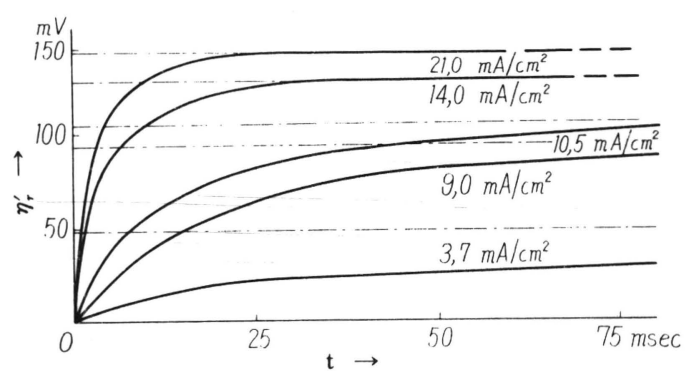

Abb. 17

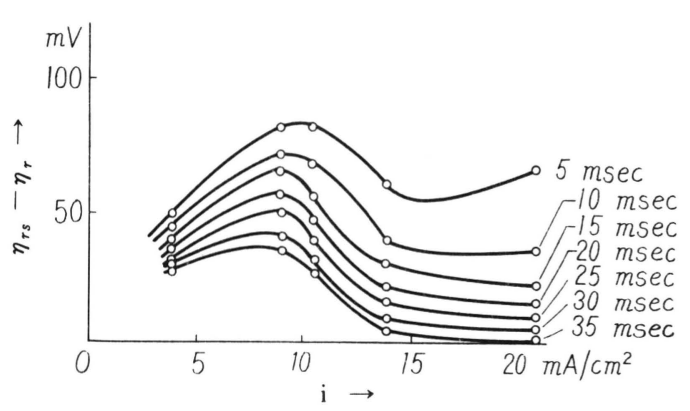

Abb. 18

aus Abb. 18 hervorgeht. Wir können damit $\eta_{w}$ eliminieren, weil man ungefähr annehmen kann, dass $\eta_{w}$ nicht nur von $t$ sondern auch von $i$ abhängig ist.

Allgemein steigen dabei diese Kurven bei kleinerer Stromdichte und beginnen dann abzufallen. Dies wiederholt sich etwas undeutlicher noch einmal und endlich findet man sonderbarer Weise, daß diese Kurve einmal unter die Null-Linie sinkt, wenn die Kurve für nach höhere Stromdichten fortführt wurde. Bei kleinerer Zeit war der Einfluß der in G1.(11-6) gegebenen sog. Doppelschichtkapazität zwar bedenklich, aber die Kurvenform ist im wesentlichen unabhängig von des Zeit. Wir müssen nun möglichst genau unterscheiden, welcher Kurventeil, nämlich das Steigen oder der Abfall der Kurve, wesentlich der Re aktionspolarisation zuzuordnen ist. Die Widerstandspolarisation hat ihre Ursache im Aufbau einer Deckschicht, die im Ver lauf einer Electrolyse bei einer bestimmten Stromdichte aufgebaut wird. Sie entsteht daher plötzlich bei einer bestimmten Stromdichte durch die Ansammelung eines elektrochemisch abgeschiedenen Stoffs. In Abb. 19 kann man deutlich den charaktristischen Eintritt der Widerstandspolarisation bei höheren Stromdichten finden, d.h. das Potential steigt über seinen stationären Zustand und bei höheren Stromdichten hat die Potentialkurve eine Spitze.

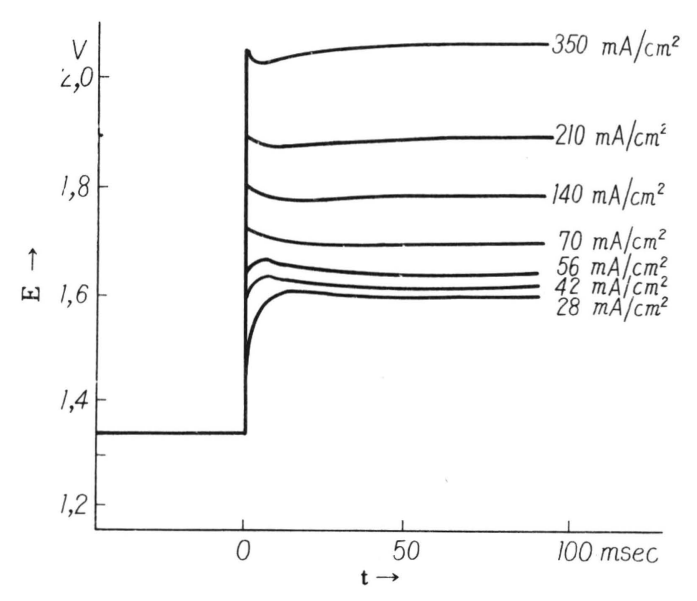

Abb. 19

Dieses Steigen bei höheren Stromdichten sofort nach dem Einschalten beruht sicher auf der Widerstands polarisation, wie Abb.

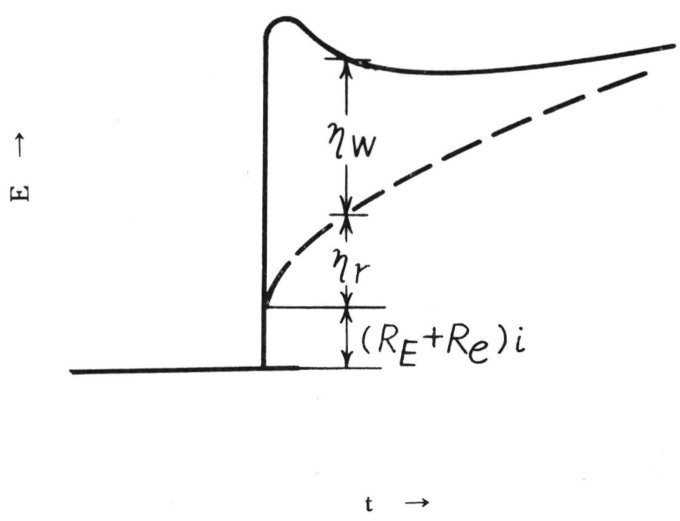

Abb. 20 
20 zeigt. Durch einen Vergleich dieser Kurven bei verschiedenen Stromdichten lassen sich jedenfalls qualitative Schlüsse hinsichtlich der Form einer Kurve reiner Reaktionspolarisation ziehen, wie Abb21 zeight.

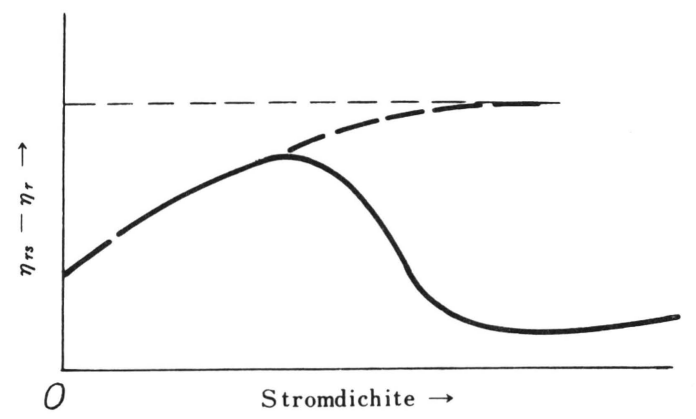

Abb. 21

Würde zuerst die Reaktionspolarisation durch den Prozess (II-i) bzw. (II-ii) bestim$\mathrm{mt}$, wobei sie mit der Gleichung

$$
\eta_{r}=\frac{R T}{n F_{r}} \ln [F(i)\{1-\exp (-k t)\}]
$$

wie in G1(5-7) bzw. G1(6-8) gezeigt, auszudrucken ist, wird die Differenz zu ihrem stationären Potential notwendigweise mit unabhängigigen Gleichung

$$
\eta_{r s}-\eta_{r}=\frac{R T}{n F_{r}} \ln \{1-\exp (-k t)\}
$$

zur Stromdichte gezeigt; es sollte sich dabei die zur $i$-Achse genau parallele Gerade handeln, so dass man den Prozess (II-i) bzw. (II-ii) offensichtlich berücksichtigen kann.

Für den Prozeß (II-iii) bzw. (II-iv) ist die Reaktionspolarisation, wie in G1(7-9) bzw. G1(8-10) gegeben, auf die Gestalt

$$
\begin{aligned}
\eta_{r} & =\frac{R T}{n_{1} F_{r}} \ln \left[F_{1}(i)\left\{1-\exp \left(-k_{1} t\right)\right\}\right] \\
& +\frac{R T}{n_{2} F_{r}} \ln \left[F_{2}(i)\left\{1-\exp \left(-k_{2} t\right)\right\}\right]
\end{aligned}
$$

reduziert. Hieraus geht in ähnlicher Weise hervor, daß der Prozeß (II-iii) bzw. (II-iv) auch keine Übereinstimmung mit der experimentalen Kurven zeigt, weil

$$
\begin{gathered}
\eta_{r s}-\eta_{r}=\frac{R T}{\pi_{1} F_{r}} \ln \left\{1-\exp \left(-k_{1} t\right)\right\} \\
+\frac{R T}{n_{2} F_{r}} \ln \left\{1-\exp \left(-k_{2} t\right)\right\}
\end{gathered}
$$

bei verschiedenen Stromdichten immer konstant ist.

Dem Prozeß (II-v) bzw. (II-vi) wird durch die Bezeichnung, wie in Gl(9-12) bzw. Gl (10-13) ausgedrückt,

$$
\begin{aligned}
\eta_{r} & =\frac{R T}{n_{1} F_{r}} \ln \left[F_{1}(i)\left\{1-\exp \left(-k_{1} t\right)\right\}\right] \\
& +\frac{R T}{n_{2} F_{r}}\left[\operatorname { l n } \left[\frac{a i+b}{c k_{1} k_{2}}\left\{1-\exp \left(-k_{2} t\right)\right\}\right.\right. \\
& \left.\left.+\frac{a i+d}{C\left(k_{2}-k_{1}\right) k_{1}}\left\{\exp \left(-k_{2} t\right)-\exp \left(-k_{1} t\right)\right\}\right]\right]
\end{aligned}
$$

genügt. Hieraus ergitt sich

$$
\begin{aligned}
& \eta_{r s}-\eta_{r}=\frac{R T}{n_{1} F_{r}} \ln \left\{1-\exp \left(-k_{1} t\right)\right\} \\
& +\frac{R T}{n_{2} F_{r}} \ln \left[\left\{1-\exp \left(-k_{2} t\right)\right\}\right. \\
& \left.+\frac{(a i+d) k_{2}}{(a i+b)\left(k_{2}-k_{1}\right)}\left\{\exp \left(-k_{2} t\right)-\exp \left(-k_{1} t\right)\right\}\right]
\end{aligned}
$$

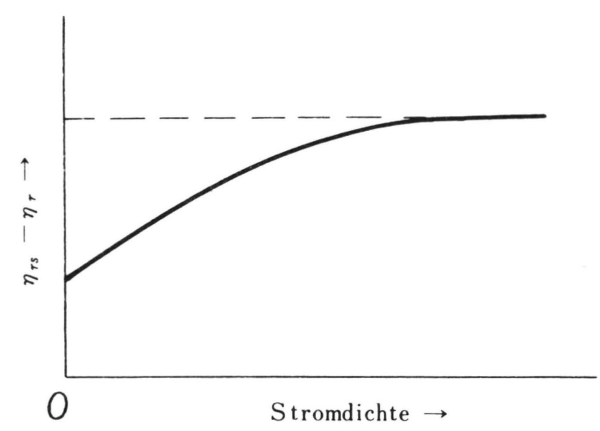

Abb. 22 
Nun läßt sich leicht nach Anhang 3 beweisen, daß diese Funktion mit der Vermehrung der Stromdichte monoton größer wird und endlich den Grenzwert erreicht, wie in Abb. 22 gezeigt, nämlich

$$
\begin{aligned}
& \left(\eta_{r s}-\eta_{r}\right)_{i=0}=\frac{R T}{n_{1} F_{r}} \ln \left\{1-\exp \left(-k_{1} t\right)\right\} \\
& +\frac{R T}{n_{2} F_{r}}\left[\ln \left\{1-\exp \left(-k_{2} t\right)\right\}\right. \\
& \left.+\frac{d k_{2}}{b\left(k_{2}-k_{1}\right)}\left\{\exp \left(-k_{2} t\right)-\exp \left(-k_{1} t\right)\right\}\right] \\
& \left(\eta_{r s}-\eta_{r}\right)_{i \rightarrow \operatorname{co}}=\frac{R T}{n_{1} F_{r}} \ln \left\{1-\exp \left(-k_{1} t\right)\right\} \\
& +\frac{R T}{n_{2} F_{r}}\left[\ln \left\{1-\exp \left(-k_{2} t\right)\right\}\right. \\
& \left.+\frac{k_{2}}{k_{2}-k_{1}}\left\{\exp \left(-k_{2} t\right)-\exp \left(-k_{1} t\right)\right\}\right]
\end{aligned}
$$

Schliesslich konnten wir hiermit die wahrscheinlichen zwei Prozesse finden. Es ist schon bekannt, dass Geschrrindigkeitskonstante nach rechts in den Reaktion

$$
\mathrm{C}+\mathrm{CO}_{2} \rightleftarrows 2 \mathrm{CO}
$$

bei $800^{\circ} \mathrm{C}-900^{\circ} \mathrm{C}$ im Vergleich zu der andren Richtung noch ziemlich klein ist, aber mit dem Steigen der Temperatur schnell gröBer wird ${ }^{15)}$. Die Tatsache, daB der CO-Gehalt im entwickelten Anodengas mit der Temperatur der Elektrolyse schnell zunimmt ${ }^{12}$, lässt darauf schliessen, dass in der Anodenreaktion diese Reaktion eine grosse Rolle spielt.

Nun müssen wir weiter darüber diskutieren, welcher Proze $B$ wirklich zu bevorzugen ist. Bei der chemischen Analyse des bei $870^{\circ} \mathrm{C}$ entwickelten Anodengases haben wir darin nur sehr wenig -- eine bestimmt zu vernachlässigende Menge -- CO gefunden. Aus diesem Resultat folgt, daß bei Prozeß (II-v) die zweite Reaktion $\left(\mathrm{CO}_{2}\right.$ $\rightarrow \mathrm{CO})$ kaum nach rechts geht, und gleichzeitig, daß bei Prozeß (II-vi) die zweite Reaktion $\left(\mathrm{CO} \rightarrow \mathrm{CO}_{2}\right)$ im Vergleich zu ihrer Rückreaktion auch nach rechts sehr schnell geht. Man findet also daß für Proze $\beta$ (II-vi) G1(10-13) sich ohne Widerspruch anwenden lä $ß t$ aber für den Prozeß (II-v) G1(6-3) statt G1(9-12) verwendet werden soll. Nachdem der Prozeß (II-v) damit ausgeschlossen wurde, kann nur der Prozeß (II-vi) als gesuchter reaktionspolarisationsbestimmender Vorgang ausgewählt werden.

\section{Widerstandspolarisation}

Es wurde schon geschrieben, daß die auftretende Widerstandspolarisation bei der Elektrolyse der Tonerde die Bestimmung der Diffusionskonstante bzw. der Diffusionsschicht und der Reaktionsprozesse stark stört. In der Nahe von ca. $25 \mathrm{~mA} / \mathrm{cm}^{2}$ ist sie eine besonders potentialbestimmende Kraft. Ihr Einfluß wurde auf den nach verschiedenen Methoden gemessenen stationären Polarisation-Stromdichte-Kurven reproduzierbar beobachtet, wie in Abb 23 wieder gegeben.

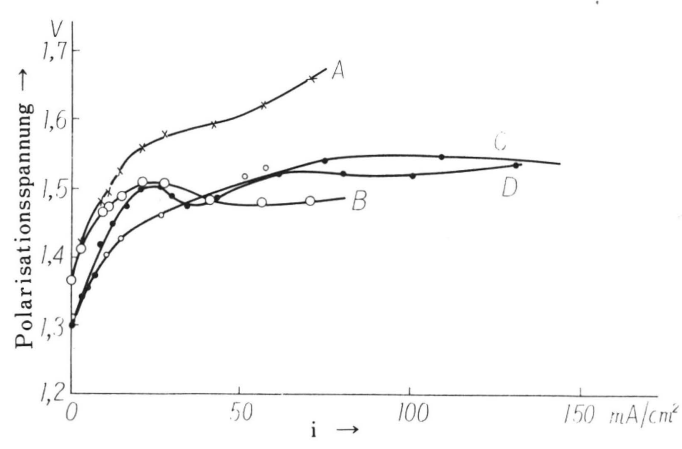

Abb. 23

Kurve A entsprichtdem mit der a galvanostatischen Methode gemessenen stationären Potential, und Kurve $B$ ist durch Abziehen der Ohmschen Spannung aus Kurve A zu bekommen. Kurve C und Kurve D wurden durch die wesentliche stationäre Messmethode bestimmt, und zwar Kurve $\mathrm{C}$ mit der 
Drossbachschen Methode (Die Widerstans-polarisation ist hier bereits mit der Ohmsche Spannung abgezogen) und Kurve D unter der Annahme konstanter Ohmscher Spannung mit einer Hilfselektrode gemessen.

Anhang 1. Setzt man zuerst G1(8-1) in G1(8--2) und G1(8-3) ein, so erhält man

$$
\frac{d C_{\mathrm{co}}}{d t}=2 k_{\mathrm{co}} C_{\mathrm{o}}-k_{\mathrm{co}}^{\prime} C_{\mathrm{co}}+\frac{d C_{\mathrm{o}}}{d t}-i \gamma+\lambda_{\mathrm{co}} \quad \frac{d C_{\mathrm{co}_{2}}}{d t}=-k_{\mathrm{co}} C_{\mathrm{o}}-k_{\mathrm{co}_{2}}^{\prime} C_{\mathrm{co}_{2}}-\frac{d C_{\mathrm{o}}}{d t}+i \gamma+\lambda \mathrm{co}_{2}
$$

Wendet man für $\mathrm{Gl}(\mathrm{A1}-1)$ und $\mathrm{Gl}(\mathrm{A1}-2)$ die Laplace-Transformation (mit den Anfangsbedin gungen) $\mathrm{G} 1(8-4)$ an, so ergibt sich

$$
L\left\{C_{\mathrm{co}}\right\}=\frac{\lambda_{\mathrm{co}}-i \gamma}{p\left(p+k_{\mathrm{co}}^{\prime}\right)}+\frac{p+2 k_{\mathrm{co}}}{p+k_{\mathrm{co}}^{\prime}} \cdot L\left\{C_{o}\right\} \quad L\left\{C_{\mathrm{co}_{2}}\right\}=\frac{\lambda_{\mathrm{co}_{2}}+i \gamma}{p\left(p+k_{\mathrm{co}_{2}}^{\prime}\right)}-\frac{p+k_{\mathrm{co}}}{p+k_{\mathrm{co}_{2}}^{\prime}} \cdot L\left\{C_{\mathrm{o}}\right\}
$$

führt man dann das Umkehrintegral durch, so erhält man

$$
\begin{aligned}
& C_{\mathrm{co}}=\frac{\left(\lambda_{\mathrm{co}}-i \gamma\right)\left\{1-\exp \left(-k_{\mathrm{co}}^{\prime} t\right)\right\}}{k_{\mathrm{co}}^{\prime}}+C_{\mathrm{o}}+\left(2 k_{\mathrm{co}}-k_{\mathrm{co}}^{\prime}\right) \exp \left(k_{\mathrm{co}}^{\prime} t\right) \int_{\mathrm{o}}^{t} C_{\mathrm{o}} \cdot \exp \left(-k_{\mathrm{co}}^{\prime} t\right) d t \\
& C_{\mathrm{co}_{2}}=\frac{\left(\lambda_{\mathrm{co}_{2}}+i \gamma\right)\left\{1-\exp \left(-k_{\mathrm{Co}_{2}}^{\prime} t\right)\right\}}{k^{\prime}}-C_{\mathrm{o}}-\left(k_{\mathrm{co}}-k_{\mathrm{Co}_{2}}\right) \exp \left(k_{\mathrm{Co}_{2}}^{\prime} t\right) \int_{\mathrm{o}}^{t} C_{\mathrm{o}} \exp _{\Gamma}\left(-k_{\mathrm{Co}_{2}}^{\prime} t\right) d t
\end{aligned}
$$

Besonders für $k_{\mathrm{co}}, k_{\mathrm{co}_{2}} \rightarrow \infty$

$$
\begin{aligned}
& C_{\mathrm{co}}=\frac{\left(\lambda_{\mathrm{co}}-i \gamma\right)\left\{1-\exp \left(-k_{\mathrm{co}}^{\prime} t\right)\right\}}{k_{\mathrm{co}}^{\prime}}+\lim _{k_{\mathrm{co}} k_{\mathrm{co}_{2} \rightarrow \infty}} C_{\mathrm{o}} \\
& +\exp \left(k_{\mathrm{co}}^{\prime} t\right) \int_{o}^{t}\left[l_{k_{\mathrm{co}} k_{\mathrm{co}_{2} \rightarrow \infty}}^{l i m}\left\{\left(2 k_{\mathrm{co}}-k_{\mathrm{co}}^{\prime}\right) C_{\mathrm{o}}\right\}\right] \exp \left(-k_{\mathrm{co}}^{\prime} t\right) d t \\
& C_{\mathrm{co}_{2}}=\frac{\left(\lambda_{\mathrm{co}_{2}}+i \gamma\right)\left\{1-\exp \left(-k_{\mathrm{co}_{2}}^{\prime} t\right)\right\}}{k_{\mathrm{CO}_{2}}^{\prime}}-\lim _{k_{\mathrm{co}} k_{\mathrm{co}_{2} \rightarrow \infty}} C_{\mathrm{o}} \\
& -\exp \left(k_{\mathrm{CO}_{2}}^{\prime} t\right) \int_{0}^{t}\left[\lim _{k_{\mathrm{co}}, k_{\mathrm{co}_{2}} \rightarrow \infty}\left\{\left(k_{\mathrm{co}^{-}}-k_{\mathrm{Co}_{2}}^{\prime}\right) C_{\mathrm{o}}\right\}\right] \exp \left(-k_{\mathrm{Co}_{2}}^{\prime} t\right) d t
\end{aligned}
$$

Es scheint die Anwendung der Reihenentwicklungsmethode am besten zu sein, um diese nichtlineare Differentialgleichung genau zu lösen. Jedoch wird die fortgesetzte Naherungsmethode trotz mathematisches Zweifels genügend die ungefähre Eigenschaft der Funktıon erkennen lassen.

Zuerst kann man einfach aus $\mathrm{Gl}(8-1)$ und $\mathrm{Gl}(3-2)$ die stationären Lösungen $U_{\mathrm{o}}$ und $U_{\text {co }}$ von $C_{o}$ und $C_{\text {co }}$ erhalten, nämlich

$$
U_{o}=\frac{2 k_{\mathrm{co}}^{\prime} i \gamma}{k_{\mathrm{co}_{n}} \lambda_{\mathrm{co}}-k_{\mathrm{co}}^{\prime} k_{\mathrm{co}}^{\prime}-k_{\mathrm{co}_{2}} i \gamma+\sqrt{\left(k_{\mathrm{co}_{2}} \lambda_{\mathrm{co}}-k_{\mathrm{co}} k_{\mathrm{co}}-k_{\mathrm{co}_{2}} i \gamma\right)^{2}+4 k_{\mathrm{co}} k_{\mathrm{co}}^{\prime} k_{\mathrm{co}_{2}}\left(\lambda_{\mathrm{co}}+i \gamma\right)}}
$$




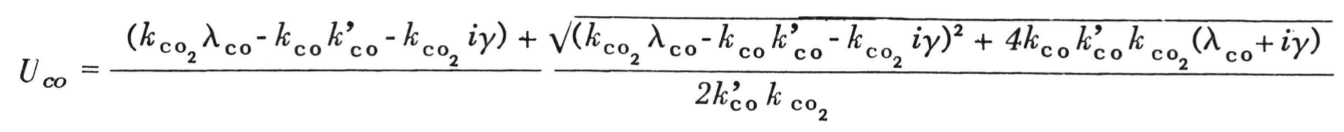

Setzt man

$$
C_{\mathrm{o}}=U_{\mathrm{o}}-Z_{\mathrm{o}}(t) \quad C_{\mathrm{co}}=U_{\mathrm{co}}-Z_{\mathrm{co}}(t)
$$

so befindet man sich praktisch im Gebiet der Werte von gemesstem $t$

$$
U_{\mathrm{o}} \geqq Z_{\mathrm{o}}(t) \geqq 0 \text { und } U_{\mathrm{co}} \geqq Z_{\mathrm{co}}(t) \geqq 0
$$

Allgemein tritt der groBe EinfluB der sog. Doppelschichtkapazität, wie in III berechnet, bei kleinem $t$ ein, deshalb hat es praktisch keinen Sinn, die genaue Konzentration für kleine $t$ auszurechnen. Nimmt man jetzt an, daB $C_{o}$ seinen stationären Zustand schnell erreicht bevor der Nonfaradaysche Strom vernachlässigt werden kann, kann man annehmen, da $\beta$ praktisch immer sehr klein ist. Diese Annahme ist wahrscheinlich möglich, weil $k_{\text {co }}$ und $k_{\mathrm{CO}_{2}}$ in Vergleich zu andren Konstanten außerordentlich groB sind. In diesem Fall ist

$$
\frac{\frac{Z_{o}}{U_{o}} \frac{Z_{c o}}{U_{c o}}}{\frac{Z_{o}}{U_{c o}}+\frac{Z_{c o}}{U_{c o}}} \ll 1
$$

Folglich ergibt sich aus $\mathrm{Gl}(8-1)$ und $\mathrm{Gl}(8-2)$

$$
\begin{gathered}
\frac{d Z_{\mathrm{o}}}{d t}=-k_{\mathrm{co}} Z_{\mathrm{o}}-k_{\mathrm{co}_{2}}\left(U_{\mathrm{co}} Z_{\mathrm{o}}+U_{\mathrm{o}} Z_{\mathrm{co}}\right) \\
\frac{d Z_{\mathrm{co}}}{d t}=k_{\mathrm{co}} Z_{\mathrm{o}}-k_{\mathrm{co}_{2}}\left(U_{\mathrm{co}} Z_{\mathrm{o}}+U_{\mathrm{o}} Z_{\mathrm{co}}\right)-k_{\mathrm{co}}^{\prime} Z_{\mathrm{co}}
\end{gathered}
$$

Damit finden wir

$$
Z_{\mathrm{o}}=\frac{(P+Q) \exp (Q t)-(P+R) \exp (R t)}{Q-R}
$$

wenn

$P=k_{\mathrm{co}}^{\prime}+k_{\mathrm{co}_{2}}\left(U_{\mathrm{o}}-U_{\mathrm{co}}\right)$

$Q=\frac{k_{\mathrm{co}}+k_{\mathrm{co}}^{\prime}+k_{\mathrm{co}}\left(U_{\mathrm{o}}+U_{\mathrm{co}}\right)+\sqrt{\left\{k_{\mathrm{co}}+k_{\mathrm{co}}^{\prime}+k_{\mathrm{co}}\left(U_{\mathrm{o}}+U_{\mathrm{co}}\right)\right\}^{2}-4\left\{k_{\mathrm{co}} k_{\mathrm{co}}^{\prime}+2 k_{\mathrm{co}} k_{\mathrm{co} O} U_{0}+k_{\mathrm{co}}^{\prime} k_{\mathrm{co}}-U_{\mathrm{co}} \underline{\}}\right.}}{2}$

$R=\frac{k_{\mathrm{co}}+k_{\mathrm{co}}^{\prime}+k_{\mathrm{co}}\left(U_{\mathrm{o}}+U_{\mathrm{co}}\right)-\sqrt{\left\{k_{\mathrm{co}}+k_{\mathrm{co}}^{\prime}+k_{\mathrm{co}}\left(U_{\mathrm{o}}+U_{\mathrm{co}}\right)\right\}^{2}-4\left\{k_{\mathrm{co}} k_{\mathrm{co}}^{\prime}+2 k_{\mathrm{co}_{0}} k_{\mathrm{co}_{2}} U_{\mathrm{o}}+k_{\mathrm{co}^{\prime}}^{\prime} k_{\mathrm{co}_{2}} U_{\mathrm{co}}\right\}}}{2}$ 
Jetzt berechnen wir die Werte für $k_{\text {co }}, k_{\mathrm{co}_{2}} \rightarrow \infty$

$$
\begin{gathered}
U_{\mathrm{o}}=0 \quad \frac{P+Q}{Q-R}=\frac{1}{1+\alpha} \quad \frac{P+Q}{Q-R}=\frac{-a}{1+\alpha} \quad P=-\infty \quad \because=-\infty \\
a=-\frac{\lambda_{\mathrm{co}}-k_{\mathrm{co}}^{\prime}-i \gamma+\sqrt{\left(\lambda_{\mathrm{coo}}-k_{\mathrm{co}}^{\prime}-i \gamma\right)^{2}+4 k_{\mathrm{co}}\left(\lambda_{\mathrm{co}}+i \gamma\right)}}{2 k_{\mathrm{co}}^{\prime}}
\end{gathered}
$$

so daß wir als kleine Funktion höherer Ordnung im Vergleich zu $U_{0}$

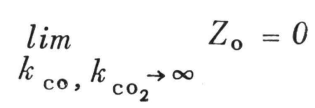

erhalten, woraus man den Beweis der obigen Annahme findet. Weiter folgt aus obigem Resultat für $k_{\mathrm{co}}, k_{\mathrm{co}_{2} \rightarrow \infty}$

$$
\begin{aligned}
& C_{o}=0 \\
& k_{\mathrm{co}} C_{\mathrm{o}}=\frac{2 k_{\mathrm{co}}^{\prime} i \gamma}{\lambda_{\mathrm{co}}+k_{\mathrm{co}}^{\prime}-i \gamma+\sqrt{\left(\lambda_{\mathrm{co}}+k_{\mathrm{co}}^{\prime}-i \gamma\right)^{2}+4 k_{\mathrm{co}}^{\prime}\left(\lambda_{\mathrm{co}}+i \gamma\right)}}
\end{aligned}
$$

Schließlich ergibt sich aus $\mathrm{Gl}(\mathrm{A2}-7)$ und $\mathrm{Gl}(\mathrm{A2}-8)$

$C_{\text {co }}=$

$$
\begin{gathered}
\frac{(i \gamma)^{2}+i \gamma\left(3 k_{\mathrm{co}}^{\prime}-2 \lambda_{\mathrm{co}}\right)+\lambda_{\mathrm{co}}\left(\lambda_{\mathrm{co}}+k_{\mathrm{co}}^{\prime}\right)+\left(\lambda_{\mathrm{co}}-i \gamma\right) \sqrt{\left(\lambda_{\mathrm{co}}+k_{\mathrm{co}}^{\prime}-i \gamma\right)^{2}+4 k_{\mathrm{co}}^{\prime}\left(\lambda_{\mathrm{co}}+i \gamma\right)}}{k_{\mathrm{co}}^{\prime}\left\{\lambda_{\mathrm{co}}+k_{\mathrm{co}}^{\prime}-i \gamma+\sqrt{\left(\lambda_{\mathrm{co}}+k_{\mathrm{co}}^{\prime}-i \gamma\right)^{2}+4 k_{\mathrm{co}}^{\prime}\left(\lambda_{\mathrm{co}}+i \gamma\right)}\right\}}\left\{1-\exp \left(-k_{\mathrm{co}_{2}}^{\prime} t\right)\right\} \\
C_{\mathrm{co}_{2}}=-\frac{-(i \gamma)^{2}+i \gamma\left(\lambda_{\mathrm{co}}-\lambda \lambda_{\mathrm{co}_{2}}+3 k_{\mathrm{co}}^{\prime}\right)+\lambda_{\mathrm{co}_{2}}\left(\lambda_{\mathrm{co}}+k_{\mathrm{co}}^{\prime}\right)+\left(\lambda \mathrm{co}_{2}+i \gamma\right) \sqrt{\left(\lambda_{\mathrm{co}}+k_{\mathrm{co}}^{\prime}-i \gamma\right)^{2}+4 k_{\mathrm{co}}^{\prime}\left(\lambda_{\mathrm{co}}+i \gamma\right)}}{k_{\mathrm{co}_{2}}^{\prime}\left\{\lambda_{\mathrm{co}}+k_{\mathrm{co}}^{\prime}-i \gamma+\sqrt{\left(\lambda_{\mathrm{co}}+k_{\mathrm{co}}^{\prime}-i \gamma\right)^{2}+4 k_{\mathrm{co}}^{\prime}\left(\lambda_{\mathrm{co}}+i \gamma\right)}\right\}} \\
\times\left\{1-\exp \left(-k_{\mathrm{co}_{2}}^{\prime} t\right)\right\} \quad(\mathrm{A} 1-13)
\end{gathered}
$$

Anhang 2. Weil G1(9-1) selbstverständlich genau gleich zu G1(6-1), so ist das Glied von $C_{o}$ in $\mathrm{Gl}(9-2)$ und $\mathrm{G} 1(9-3)$ in ähnlicher Weise durch $\mathrm{Gl}(6-4)$ zu eliminieren, nämlich

$$
\frac{d C_{\mathrm{Co}_{2}}}{d t}=\frac{i y}{2}+\lambda_{\mathrm{co}_{2}}-\left(k_{\mathrm{co}_{2}}^{\prime}+k_{\mathrm{co}}\right) C_{\mathrm{co}_{2}}+k_{\mathrm{co}}^{\prime m} C_{\mathrm{co}}^{2} \quad \frac{d C_{\mathrm{co}}}{d t}=\lambda_{\mathrm{co}}+2 k_{\mathrm{co}} C_{\mathrm{co}_{2}}-k_{\mathrm{co}}^{\prime} C_{\mathrm{co}}-2 k_{\mathrm{co}}^{m} C_{\mathrm{co}}^{2}
$$

Hieraus gilt sofort

$$
\frac{d}{d t}\left(C_{\mathrm{co}}+2 C_{\mathrm{co}_{2}}\right)=i \gamma+2 \lambda_{\mathrm{co}_{2}}+\lambda_{\mathrm{co}}-k,\left(\mathrm{C}_{\mathrm{co}}+2 C_{\mathrm{co}_{2}}\right)
$$

wenn man dabei annimmt, da $\beta \quad k_{\mathrm{co}}^{\prime}=k_{\mathbf{B O}_{2}}^{\prime} \equiv k$ '

$$
\text { Folglich ganz einfach } \quad C_{\mathrm{co}}+2 C_{\mathrm{co}_{2}}=\frac{i \gamma+2 \lambda_{\mathrm{co}_{2}}+\lambda}{k}
$$


Aus $\mathrm{Gl}(\mathrm{A} 2-1)$ und $\mathrm{Gl}(\mathrm{A} 2-2)$ kann man die stationäre Lösung von $C_{c o}$

$$
U_{\mathrm{co}}=\left[-k^{\prime}\left(k^{\prime}+k_{\mathrm{co}}\right)\right.
$$

$\left.+\sqrt{k^{\prime 2}\left(k^{\prime}+k_{\mathrm{co}}\right)^{2}+8 k^{\prime} k_{\mathrm{co}}^{m}\left(i \lambda+2 \lambda_{\mathrm{co}_{2}}\right)+\lambda_{\mathrm{co}}\left(k^{\prime}+k_{\mathrm{co}}\right)}\right]$

$$
/ 4 k^{\prime} k_{\text {co }}^{\prime \prime \prime}
$$

leight erhalten. Setzt man dann die Lösung von $\mathrm{C}_{\mathrm{co}}$

$$
C_{\text {co }}=U_{\text {co }}-Z_{\text {co }}(t)
$$

so gibt

$$
\frac{d Z_{\mathrm{co}}}{d t}=-A \exp \left(-k^{\prime} t\right)+B Z_{\mathrm{co}}-C Z_{\mathrm{co}}^{2}
$$

wenn

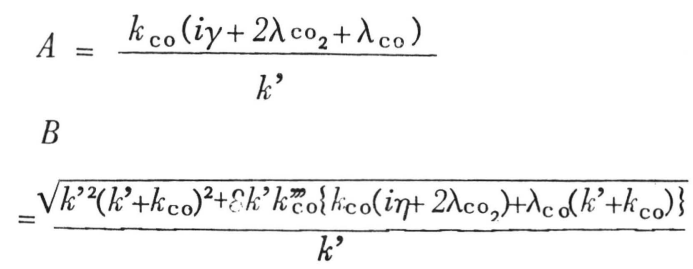

$$
C=2 k_{\mathrm{co}}^{m}
$$

Setzt man weiter

$$
\phi(t)=\exp \left(C \int Z_{\mathrm{co}} d t\right)
$$

so erhält man

$$
\frac{d^{2} \phi}{d t^{2}}-B \frac{d \phi}{d t}+A C_{\phi} \exp \left(-k^{\prime} t\right)=0
$$

weil

$$
Z_{\mathrm{co}}=\frac{\frac{d \phi}{d t}}{C_{\phi}}
$$

folglich auch mit den Anfangsbedingungen

$$
\phi(o)=1
$$

$$
\left(\frac{d \phi}{d t}\right)_{\mathrm{t}=\mathrm{o}}=C U_{\mathrm{o}}
$$

Damit konnten wir die lineare Differentialgleichung erhalten, jedoch in einer etwas unhandlichen Form, um sie unmittelbar zu lösen. Deshalb setzen wir noch einmal um

$$
\phi(t)=\psi(\xi) \text { und } \xi=\exp \left(-k^{\prime} t\right)
$$

und erhalten eine Besselsche Differentialgleichung

$$
\xi \frac{d^{2} \psi}{d \xi^{2}}+a \frac{d \psi}{d \xi}+\beta \psi=0
$$

mit den Anfang sbedingungen

$$
\begin{aligned}
& \left(\frac{d \psi}{d \xi}\right) \quad \xi=1=\frac{C U_{\mathrm{ca}}}{k^{9}} \\
& \psi(1)=1
\end{aligned}
$$

wenn

$$
d \cdot=\frac{k^{\prime}+B}{k^{\prime}} \text { und } B=\frac{A C}{k^{\prime 2}}
$$

Die Lösung dieser Differentialgleichung kann man in Ausdruck zylindrischen Form finden, nämlich

$$
\psi(\xi)=\xi^{\frac{1-\alpha}{2}} Z_{\nu}\left(2 \sqrt{\beta} \xi^{1 / 2}\right)
$$

wenn

$$
\gamma=1-\alpha
$$

weil $\nu$ sicher keine ganze Zahl ist, also gilt die Beziehung

$$
Z_{\nu}=C_{1} J_{\nu}+C_{2} J_{-\nu}
$$


wobei $C_{1}$ und $C_{2}$ Konstanten sind. Aus den Anfangsbedingungen ergibt sich

$$
\begin{aligned}
& C_{1}= \\
& \frac{\left(k^{\prime}+k_{\mathrm{co}^{-}}-B\right) J_{-\nu}(2 \sqrt{\bar{\beta}})+k^{\prime} \sqrt{\beta} J_{-\nu+1}(2 \sqrt{\bar{\beta}})}{k^{\prime} \Delta} \\
& C_{\mathbf{2}}=\frac{-\left(k^{\prime}+k_{\mathrm{co}}+B\right) J_{\nu}(2 \sqrt{\beta})-k^{\prime} \sqrt{\beta J} J_{\nu+1}(2 \sqrt{\beta})}{k^{\prime} \Delta} \\
& \Delta=J_{\nu}(2 \sqrt{\beta})\left[\nu J_{-\nu}(2 \sqrt{\beta})\right. \\
& \left.+2 \sqrt{\beta} J_{-\nu+1}(2 \sqrt{\beta})\right]+J_{-\nu}(2 \sqrt{ } \beta)\left[\nu J_{\nu}\right. \\
& \left.\cdot(2 \sqrt{\beta})-2 \sqrt{\beta} J_{\nu+1}(2 \sqrt{\beta})\right]
\end{aligned}
$$$$
(A 2-13)
$$

Anhang 3.

Es ist klar, da $\beta$ in $\mathrm{Gl}(12-11)$ des erste logarithmische Glied, das auch im zwieten logarithmische Glied nochmal enthalten ist, von $i$ abhängig ist. Wir bezeichnen den Rest des 2. logarithmischen Gliedes mit $\phi(i)$, nämlich

$$
\phi(i)=\frac{a i+d}{a i+b} \frac{k_{2}}{k_{2}-k_{1}}\left\{\exp \left(-k_{2} t\right)-\exp \left(-k_{1} t\right)\right\}
$$

wenn $k_{1}>k_{2}$, so gilt offenbar

$$
\phi>0
$$

und

$$
\frac{d \phi}{d t}>0
$$

weil aus $\mathrm{Gl}(9-12)$ oder $\mathrm{Gl}(10-13)$ b>d ist. Wenn $\mathrm{K}_{2}<\mathrm{K}_{1}$, so gilt auch

$$
\phi>0
$$

und

$$
\frac{d \phi}{d i}>0
$$

\section{Literatur:}

1) G. Kortum, Lehrbuch der Elektrochemie, 476 (1957) Weinheim Bergstr.

2) G. Eger, Handbuch der Technischen Elektrochemie, Bd. III, 2. Aufl. (1955) Leipzig

3) P. Drossbach, T. Hashino, P. Krahl, W. Peiffer, Chem. Ing. Tech. 33, 84 (1961)

4) L.M. Foster u. W.B. Frank, J. Phys. Chem. 61, 1531 (1957)

5) L.M. Foster u. W.B. Frank, J. Electrochem. Soc. 107, 997 (1960)

6) F. Bonnier, Bull. Soc. Chim. France Mém., DI-DII 5, 21 (1954)

7) K. Resch, Z. Erz. u. Metallh., XIII 523 (1960)

8) C.A. Knorr, Z. Elektrnchem., 59647 (1955)

9) T.R. Rosebruch und W. Lash Miller, J. Phys. Chem. 14816 (1910)

10) P. Drossbach, Grundriss der allgem. tech. Electrochem., 1, 251 (1952) Berlin

11) R. Piontelli, Chim。 e Indo, 22501 (1940)

12) T.G. Peason u. J. Waddington, Discuss. Faraday Soc., 1, 307 (1947)

13) R. Gadeau, J. Four. electr., 57,8 (1948)

14) J.E. Boner, Helvetica Chem. Acta, 33, 1137 (1950)

15) Zusammenfassende Darstellung: Der Chemie Ingenieur, Bd. III 251 Herausgegeben von A. Eucken 